\title{
Decay Estimates for Hyperbolic Balance Laws
}

\author{
Shuichi Kawashima and Wen-An Yong
}

\begin{abstract}
This work is concerned with time-decay properties of small-amplitude global smooth solutions to the initial value problem for hyperbolic systems of balance laws admitting an entropy and satisfying the stability condition. By using energy methods in both the physical space and the Fourier space, we obtain the optimal decay estimates of solutions and their derivatives in the $L^{2}$-norm up to order $s-1$, provided that the initial data are in $H^{s}$. A key ingredient in our analysis is a timeweighted energy estimate, leading to a decay estimate for multi-dimensional problems without assuming the $L^{1}$ property on initial data.
\end{abstract}

Keywords. Hyperbolic balance laws, entropy, stability condition, global smooth solutions, decay estimates

Mathematics Subject Classification (2000). Primary 35L45, secondary 35B40, $82 \mathrm{~B} 35$

\section{Introduction}

Hyperbolic balance laws are partial differential equations of the form

$$
w_{t}+\sum_{j=1}^{d} f^{j}(w)_{x_{j}}=g(w) .
$$

Here $w=w(x, t)$ is the unknown $m$-vector valued function of spatial variable $x=\left(x_{1}, x_{2}, \ldots, x_{d}\right) \in \mathbb{R}^{d}$ and time $t \geq 0$, taking values in an open convex set $\mathcal{O}_{w} \subset \mathbb{R}^{m}$ (called state space); $f^{j}(w)(j=1,2, \ldots, d)$ and $g(w)$ are given smooth mappings from $\mathcal{O}_{w}$ into $\mathbb{R}^{m}$; and the subscripts $t$ and $x_{j}$ refer to the partial derivatives with respect to $t$ and $x_{j}$, respectively.

As fundamental partial differential equations and as transition models between the Boltzmann equation and hyperbolic conservation laws, balance laws (1.1) describe a great number of non-equilibrium phenomena. Important

Shuichi Kawashima: Graduate School of Mathematics, Kyushu University, Fukuoka 812-8581, Japan; kawashim@math.kyushu-u.ac.jp

Wen-An Yong: Zhou Pei-Yuan Center for Appl. Math., Tsinghua University, Beijing 100084, China; wayong@tsinghua.edu.cn 
examples occur in the study of chemically reactive flows, gas dynamics with relaxation, radiation hydrodynamics, traffic flows, nonlinear optics, and so on. See [14] and references cited therein.

Consider initial value problems of (1.1) with initial data close to a constant state in equilibrium. We know that the existence of global smooth solutions to the above problems was first pointed out in [14] for the general system (1.1) satisfying an entropy-dissipation condition therein and the stability condition introduced in [12]. The result can be regarded as a generalization of that in $[7,12]$ for discrete-velocity kinetic models and can be proved with exactly the same argument as in $[7,12]$. The details were later carried out in [3] for onedimensional problems and in [15] for multi-dimensional ones. In addition, a quite different approach was used in [13] to show the global existence for a specific model - three-dimensional compressible Euler equations with damping. Moreover, some time-decay properties and the Chapman-Enskog limits of the global solutions were earlier obtained in $[7,8]$ for discrete-velocity kinetic models and in $[1,16]$ for the general system (1.1) satisfying the above entropy-dissipation and stability conditions.

In this paper, we intend to improve the main decay estimates in $[1,16]$ and thus generalize the results in $[7,8]$ for discrete-velocity kinetic models - a class of semilinear problems - to the general quasilinear system (1.1). We assume that the general system (1.1) admits an entropy (function) defined in [9] and satisfies the stability condition in [12]. The main difficulty of this work stems from the quasilinearity of (1.1) and arises when deriving decay estimates for derivatives of the solution. To overcome this difficulty, we employ a time-weighted energy method which was first developed in [11] for the compressible Navier-Stokes equation (see also [5]).

As a by-product, the time-weighted energy estimates lead to a decay estimate for the multi-dimensional problems without assuming the $L^{1}$-smallness of initial data. Namely,

Theorem 1.1. Let $d \geq 2$ and $s>\frac{d}{2}+1$ an integer. Fix $\bar{w} \in \mathcal{O}_{w}$ satisfying $g(\bar{w})=0$ and suppose $w_{0}=w_{0}(x)$ is close sufficiently to $\bar{w}$ in the usual Sobolev space $H^{s}=H^{s}\left(\mathbb{R}^{d}\right)$. Then, under the above entropy and stability conditions, the global-in-time solution $w=w(x, t)$ to (1.1) with initial data $w_{0}=w_{0}(x)$ satisfies the following decay estimates

$$
\left\|\partial_{x}^{k}(w(\cdot, t)-\bar{w})\right\|_{L^{p}} \leq C\left\|w_{0}-\bar{w}\right\|_{H^{s}}(1+t)^{-\gamma_{p}^{\prime}-\frac{k}{2}}
$$

for $p \in[2, \infty]$ and $0 \leq k \leq s-2 \gamma_{p}^{\prime}\left(k \neq s-\frac{d}{2}\right.$ if $\left.p=\infty\right)$, where $\gamma_{p}^{\prime}=\frac{d}{2}\left(\frac{1}{2}-\frac{1}{p}\right)$ is the decay exponent of the $L^{p}-L^{2}$ decay estimate for the linear heat equation.

In (1.2), $C$ is a generic constant and other notation are explained in the end of this section. Remark that $\gamma_{2}^{\prime}=0$ for $p=2$ and that Theorem 1.1 does 
not assume that $w_{0}(x)-\bar{w} \in L^{1}\left(\mathbb{R}^{d}\right)$ and thus is not contained in $[1,16]$. The complete version of this theorem is Theorem 4.3 in Section 4 with further details and conclusions.

Having the time-weighted estimates, we adopt the standard linearization method, also used in [1], to derive sharper decay estimates under the assumption that the initial data are close to the constant state in the $L^{1}$-norm. This approach requires suitable decay properties of the corresponding linearized problems. The required properties can be derived with the energy method in the Fourier space, while one-dimensional problems need an additional spectral analysis of the linearized operator. Moreover, we use a technique from [7], instead of the Duhamel principle, for multi-dimensional problems. The main results are stated in Theorem 6.2 for multi-dimensional problems and in Theorem 7.3 for one-dimensional ones. They can briefly summarized as follows.

Theorem 1.2. Let $s>\frac{d}{2}+1$. Suppose $w_{0}-\bar{w} \in H^{s} \cap L^{1}$ and

$$
E_{1}:=\left\|w_{0}-\bar{w}\right\|_{H^{s}}+\left\|w_{0}-\bar{w}\right\|_{L^{1}}
$$

is sufficiently small. Then, under the above entropy and stability conditions, the global solution $w(x, t)$ to (1.1) satisfies the following decay estimates

$$
\left\|\partial_{x}^{k}(w(\cdot, t)-\bar{w})\right\|_{L^{p}} \leq C E_{1}(1+t)^{-\gamma_{p}-\frac{k}{2}}
$$

for $p \in[2, \infty]$ and $0 \leq k \leq s-1-2 \gamma_{p}^{\prime}\left(k \neq s-1-\frac{d}{2}\right.$ if $\left.p=\infty\right)$. Here $\gamma_{p}=\frac{d}{2}\left(1-\frac{1}{p}\right)$ is the decay exponent of the $L^{p}-L^{1}$ decay estimate for the linear heat equation and $\gamma_{p}^{\prime}$ is the same as in Theorem 1.1 .

Besides the aforementioned works $[7,8,12]$, let us mention that the decay estimate (1.3) with $k=0$ was earlier derived in [16]. Recently, similar decay estimates have been obtained by Bianchini et al. in [1]. The authors did not use our time-weighted energy estimates to treat the difficulty due to the quasilinearity, and consequently they derived (1.3) only for non-negative integer $k$ with $\sigma(=s-k$ ) large enough (see the theorems in Section 5 of [1]).

Moreover, our method is different from that used in [1], where a somewhat long analysis of the Green function for the linearized system is used to derive decay properties of the corresponding linearized problems. In contrast, the present paper derives the required properties by using the energy method in the Fourier space, while one-dimensional problems need an additional spectral analysis of the linearized operator. Moreover, we use a technique from [7], instead of the Duhamel principle, for multi-dimensional problems.

The paper is organized as follows. In Section 2 we review the notion of entropy and the stability condition for (1.1) from [9] and [12], respectively. The energy method is recalled in Section 3 to show the existence of smallamplitude global smooth solutions under the framework of [9]. Section 4 is 
devoted to the time-weighted energy estimates. In Section 5, we use the energy method in the Fourier space to derive the optimal decay estimate for the corresponding linearized problems. The sharper decay estimates for nonlinear problems are derived in Section 6 for multi-dimensional case and in Section 7 for one-dimensional one. Finally, in Appendix we analyze spectral properties of one-dimensional operators in the low-frequency region.

Notation. We use $\langle\cdot, \cdot\rangle$ and $(\cdot, \cdot)$ to denote the standard inner products in the real $\mathbb{R}^{m}$ and in the complex $\mathbb{C}^{m}$, respectively. $|\cdot|$ is the corresponding norm in $\mathbb{R}^{m}$ or $\mathbb{C}^{m}$. For a function $u$ on $\mathbb{R}^{d}$ and a positive integer $k, \partial_{x}^{k} u$ denotes either all the $k$-th order derivatives $\left\{\partial_{x}^{\alpha} u ;|\alpha|=k\right\}$ or any one of them, where $\alpha=\left(\alpha_{1}, \alpha_{2}, \ldots, \alpha_{d}\right)$ is a multi-index. Letters $c$ and $C$ denote positive generic constants which may vary from line to line. For $p \in[1, \infty], L^{p}=L^{p}\left(\mathbb{R}^{d}\right)$ is the usual Lebesgue space on $\mathbb{R}^{d}$. For $s \geq 0$ an integer, $H^{s}=H^{s}\left(\mathbb{R}^{d}\right)$ is the Sobolev space which consists of functions in $L^{2}$ whose distribution derivatives of order $\leq s$ are all in $L^{2}$. For a Banach space $X$, we always denote its norm by $\|\cdot\|_{X}$. When $u$ depends on $t$ as well as on $x$, we write $\|u(t)\|_{X}$ or $\|u(\cdot, t)\|_{X}$ to recall that the norm is taken with respect to $x$ while $t$ is viewed as a parameter. Furthermore, $C(\mathcal{I} ; X)$ denotes the space of continuous functions on the interval $\mathcal{I}$ with values in $X$.

\section{Preliminaries}

In this section, we review the notion of entropy and the stability condition for (1.1) from [9] and [12], respectively. To begin with, we set

$$
\mathcal{M}:=\left\{\psi \in \mathbb{R}^{m} ;\langle\psi, g(w)\rangle=0 \text { for all } w \in \mathcal{O}_{w}\right\} .
$$

In the discrete kinetic theory [12], $\mathcal{M}$ is called the space of summational (collision) invariants. Moreover, we denote by $\mathcal{E}$ the set of equilibrium states for (1.1):

$$
\mathcal{E}:=\left\{w \in \mathcal{O}_{w} ; g(w)=0\right\}
$$

In the following, $D_{w}$ denotes the (row) gradient operator with respect to $w$ and the superscript $T$ denotes the transpose.

2.1. Entropy and symmetrization. In [9], an entropy (function) for (1.1) is defined as follows.

Definition 2.1. Let $\eta=\eta(w)$ be a smooth function defined on $\mathcal{O}_{w}$. It is called an entropy (function) for (1.1) if the following four conditions are satisfied:

- $\eta(w)$ is strictly convex on $\mathcal{O}_{w}$ in the sense that the Hessian $D_{w}^{2} \eta(w)$ is positive definite for $w \in \mathcal{O}_{w}$.

- The matrix $D_{w} f^{j}(w)\left(D_{w}^{2} \eta(w)\right)^{-1}$ is symmetric for $w \in \mathcal{O}_{w}$ and $j=$ $1,2, \ldots, d$. 
- Let $w \in \mathcal{O}_{w}$. Then $w \in \mathcal{E}$ if and only if $\left(D_{w} \eta(w)\right)^{T} \in \mathcal{M}$.

- For $w \in \mathcal{E}$, the matrix $D_{w} g(w)\left(D_{w}^{2} \eta(w)\right)^{-1}$ is symmetric and nonpositive definite, and its null space coincides with $\mathcal{M}$.

Let $\eta=\eta(w)$ be an entropy defined thus and put

$$
u(w):=\left(D_{w} \eta(w)\right)^{T} .
$$

It was shown in [9] that the mapping $u=u(w)$ is a diffeomorphism from $\mathcal{O}_{w}$ onto its range $\mathcal{O}_{u}$. Let $w=w(u)$ be the inverse mapping which is a diffeomorphism from $\mathcal{O}_{u}$ onto $\mathcal{O}_{w}$. Putting $w=w(u)$ in (1.1), we obtain

$$
A^{0}(u) u_{t}+\sum_{j=1}^{d} A^{j}(u) u_{x_{j}}=h(u)
$$

where

$$
\begin{aligned}
A^{0}(u) & :=D_{u} w(u) \\
A^{j}(u) & :=D_{u} f^{j}(w(u))=\left(D_{w} f^{j}\right)(w(u)) D_{u} w(u) \\
h(u) & :=g(w(u)) .
\end{aligned}
$$

Let us define

$$
L(u):=-D_{u} h(u)=-\left(D_{w} g\right)(w(u)) D_{u} w(u) .
$$

Since $D_{u} w(u)=\left(\left(D_{w}^{2} \eta\right)(w(u))\right)^{-1}$ by $(2.1)$, we see that

$$
\begin{aligned}
A^{0}(u) & :=\left(D_{w}^{2} \eta(w)\right)^{-1} \\
A^{j}(u) & :=D_{w} f^{j}(w)\left(D_{w}^{2} \eta(w)\right)^{-1} \\
L(u) & :=-D_{w} g(w)\left(D_{w}^{2} \eta(w)\right)^{-1},
\end{aligned}
$$

where $w$ on the right-hand side is evaluated at $w(u)$. Moreover, it was shown in Theorem 2.1 of [9] that (2.2) is a symmetric dissipative system in the sense defined as follows.

Definition 2.2. The system (2.2) is called symmetric dissipative if it satisfies the following four conditions:

- $A^{0}(u)$ is symmetric and positive definite for $u \in \mathcal{O}_{u}$.

- $A^{j}(u)$ is symmetric for $u \in \mathcal{O}_{u}$ and $j=1,2, \ldots, d$.

- Let $u \in \mathcal{O}_{u}$. Then $h(u)=0$ if and only if $u \in \mathcal{M}$.

- For $u \in \mathcal{M}$, the matrix $L(u)=-D_{u} h(u)$ is symmetric and nonnegative definite, and its null space coincides with $\mathcal{M}$.

Also, we know from Proposition 3.1 of [9] that $h(u)$ has the following useful expression for any fixed constant state $\bar{u} \in \mathcal{M}$ :

$$
h(u)=-L u+r(u),
$$


where $L=L(\bar{u}), r(u) \in \mathcal{M}^{\perp}$ (the orthogonal complement of $\mathcal{M}$ ) for all $u \in \mathcal{O}_{u}$ and satisfies

$$
|r(u)| \leq C|u-\bar{u}||(I-P) u|
$$

for $u \in \mathcal{O}_{u}$ close to $\bar{u}$, with $I$ the identity mapping on $\mathbb{R}^{m}$ and $P$ the orthogonal projection onto $\mathcal{M}$.

In addition, we recall the equation satisfied by the entropy $\eta(w)$. Since $A^{j}(u)=D_{u} f^{j}(w(u))$ is symmetric, it is well-known that there exists a smooth function $\tilde{q}^{j}(u)$ defined on $\mathcal{O}_{u}$ such that $\left(D_{u} \tilde{q}^{j}(u)\right)^{T}=f^{j}(w(u))$. Define

$$
q^{j}(w):=\left\langle u(w), f^{j}(w)\right\rangle-\tilde{q}^{j}(u(w)) .
$$

We know from [9] that the entropy $\eta(w)$ verifies

$$
\eta(w)_{t}+\sum_{j=1}^{d} q^{j}(w)_{x_{j}}=\langle u(w), g(w)\rangle,
$$

which can be derived by taking the inner product of (1.1) with $u(w)$ and using the fact that $\left(D_{w} q^{j}(w)\right)^{T}=\left(D_{w} f^{j}(w)\right)^{T} u(w)$.

2.2. Normal form. In [9], we also transformed (1.1) to a symmetric dissipative system of the normal form in the sense defined below.

Definition 2.3. A symmetric dissipative system (2.2) is said to be of the normal form if $A^{0}(u)$ is block-diagonal associated with the orthogonal decomposition $\mathbb{R}^{m}=\mathcal{M} \oplus \mathcal{M}^{\perp}$.

Use the partition as $w=\left(\begin{array}{l}w_{1} \\ w_{2}\end{array}\right), u=\left(\begin{array}{l}u_{1} \\ u_{2}\end{array}\right)$ associated with the orthogonal decomposition $\mathbb{R}^{m}=\mathcal{M} \oplus \mathcal{M}^{\perp}$. We consider the mapping $w \longrightarrow v$ defined by

$$
v:=\left(\begin{array}{l}
w_{1} \\
u_{2}
\end{array}\right)
$$

where $u_{2}=\left(D_{w_{2}} \eta(w)\right)^{T}$. This is a diffeomorphism from $\mathcal{O}_{w}$ onto its range $\mathcal{O}_{v}$. Denote by $w=w(v)$ the inverse mapping which is a diffeomorphism from $\mathcal{O}_{v}$ onto $\mathcal{O}_{w}$. We set $w=w(v)$ in (1.1) and multiply from the left with $D_{v} w(v)^{T}\left(D_{w}^{2} \eta\right)(w(v))$ to obtain

$$
\tilde{A}^{0}(v) v_{t}+\sum_{j=1}^{d} \tilde{A}^{j}(v) v_{x_{j}}=\tilde{h}(v),
$$

where

$$
\begin{aligned}
\tilde{A}^{0}(v) & :=\left(D_{v} w\right)^{T} D_{w}^{2} \eta(w) D_{v} w \\
\tilde{A}^{j}(v) & :=\left(D_{v} w\right)^{T} D_{w}^{2} \eta(w) D_{w} f^{j}(w) D_{v} w \\
\tilde{h}(v) & :=\left(D_{v} w\right)^{T} D_{w}^{2} \eta(w) g(w),
\end{aligned}
$$

and $w$ on the right-hand side is evaluated at $w(v)$. 
Let $u=u(v)$ be the diffeomorphism composed by $u=u(w)$ and $w=$ $w(v)$. Then a straightforward computation using the relation $\left(D_{v} w\right)^{T} D_{w}^{2} \eta(w)=$ $\left(D_{v} u\right)^{T}$ shows that

$$
\begin{aligned}
\tilde{A}^{0}(v) & :=\left(D_{v} u\right)^{T} A^{0}(u) D_{v} u \\
\tilde{A}^{j}(v) & :=\left(D_{v} u\right)^{T} A^{j}(u) D_{v} u \\
\tilde{h}(v) & :=\left(D_{v} u\right)^{T} h(u),
\end{aligned}
$$

where $A^{0}(u), A^{j}(u)$ and $h(u)$ are given in (2.3), and $u$ is evaluated at $u(v)$. This means that the system (2.9) can also be obtained from (2.2) by multiplying from the left with $\left(D_{v} u\right)^{T}$.

Theorem 3.3 of [9] indicates that (2.9) is a symmetric dissipative system of the normal form. Precisely, we have the following refinement of Theorem 3.3 of $[9]$.

Theorem 2.4. The system (2.9) is a symmetric dissipative system of the normal form and $\tilde{h}(v)=h(u)$. Moreover, between the variables $u$ and $v$ it holds that $u \in \mathcal{M}$ if and only if $v \in \mathcal{M}$. Furthermore, the matrix $\tilde{L}(v):=-D_{v} \tilde{h}(v)$ can be expressed in terms of $L(u)$ defined in (2.4) as

$$
\tilde{L}(v)=\left(D_{v} u\right)^{T} L(u) D_{v} u
$$

and verifies $\tilde{L}(v)=L(u)$ if $v \in \mathcal{M}$ (i.e., $u \in \mathcal{M})$.

Proof. We give an outline of the proof. Use the partition of $A^{0}(u)$ and $h(u)$ as

$$
A^{0}(u)=\left(\begin{array}{cc}
A_{11}^{0} & A_{12}^{0} \\
A_{21}^{0} & A_{22}^{0}
\end{array}\right), \quad h(u)=\left(\begin{array}{c}
0 \\
h_{2}
\end{array}\right)
$$

associated with the decomposition $\mathbb{R}^{m}=\mathcal{M} \oplus \mathcal{M}^{\perp}$. Then, noting (2.8) and (2.3), we have

$$
D_{u} v=\left(\begin{array}{cc}
A_{11}^{0} & A_{12}^{0} \\
O & I_{r}
\end{array}\right),
$$

where $I_{r}$ is the unit matrix of order $r:=\operatorname{dim}\left(\mathcal{M}^{\perp}\right)$, and hence

$$
D_{v} u=\left(\begin{array}{cc}
\left(A_{11}^{0}\right)^{-1} & -\left(A_{11}^{0}\right)^{-1} A_{12}^{0} \\
O & I_{r}
\end{array}\right) .
$$

Now, a straightforward computation shows that

$$
\tilde{A}^{0}(v)=\left(\begin{array}{cc}
\left(A_{11}^{0}\right)^{-1} & O \\
O & \left(\tilde{A}_{22}^{0}\right)^{-1}
\end{array}\right), \quad \tilde{h}(v)=\left(\begin{array}{c}
0 \\
h_{2}
\end{array}\right),
$$


where $\tilde{A}_{22}^{0}:=A_{22}^{0}-A_{21}^{0}\left(A_{11}^{0}\right)^{-1} A_{12}^{0}$. Therefore, $\tilde{A}^{0}(v)$ is block-diagonal and $\tilde{h}(v)=h(u)$.

Next, it follows from $\tilde{h}(v)=h(u)$ that $\tilde{L}(v)=L(u) D_{v} u$. Here $L(u)$ is defined in (2.4) and is expressed as

$$
L(u)=\left(\begin{array}{cc}
O & O \\
L_{21} & L_{22}
\end{array}\right),
$$

where $L_{21}=O$ for $u \in \mathcal{M}$ as observed in [9]. Therefore, a direct computation using (2.10) gives

$$
\tilde{L}(v)=\left(\begin{array}{cc}
O & O \\
L_{21}\left(A_{11}^{0}\right)^{-1} & \tilde{L}_{22}
\end{array}\right),
$$

where $\tilde{L}_{22}=L_{22}-L_{21}\left(A_{11}^{0}\right)^{-1} A_{12}^{0}$. Thus, we see that $\tilde{L}(v)=\left(D_{v} u\right)^{T} L(u) D_{v} u$. On the other hand, $v \in \mathcal{M}$ means that $u_{2}=0$ in (2.8), which is equivalent to $u \in \mathcal{M}$. Since $L_{21}=0$ for $u \in \mathcal{M}$, we see that $\tilde{L}(v)=L(v)$ if $u \in \mathcal{M}$ (i.e., $v \in \mathcal{M})$. The other statements are obvious and hence the proof is complete.

As a simple corollary, we have an analogue of $(2.5)$ that $\tilde{h}(v)$ has the following expression for any fixed constant state $\bar{v} \in \mathcal{M}$ (i.e., $\bar{u} \in \mathcal{M}$ ):

$$
\tilde{h}(v)=-L v+\tilde{r}(v),
$$

where $L=L(\bar{u})$ as in (2.5), and $\tilde{r}(v) \in \mathcal{M}^{\perp}$ for all $v \in \mathcal{O}_{v}$ and

$$
|\tilde{r}(v)| \leq C|v-\bar{v}||(I-P) v|
$$

for $v \in \mathcal{O}_{v}$ close to $\bar{v}$.

2.3. Stability condition. In order to show the global existence for the initial value problem of (1.1), we formulate the stability condition for the symmetric dissipative system (2.2) or (2.9) obtained from (1.1). Let $\bar{u} \in \mathcal{M}$ be a constant state and consider the linearized system of (2.2) at $u=\bar{u}$ :

$$
A^{0} u_{t}+\sum_{j=1}^{d} A^{j} u_{x_{j}}+L u=0
$$

where $A^{0}=A^{0}(\bar{u}), A^{j}=A^{j}(\bar{u})$ and $L=L(\bar{u})$. Taking the Fourier transform of (2.13) with respect to $x$, we obtain

$$
A^{0} \hat{u}_{t}+i|\xi| A(\omega) \hat{u}+L \hat{u}=0
$$

where $A(\omega):=\sum_{j=1}^{d} A^{j} \omega_{j}$ with $\omega=\frac{\xi}{|\xi|} \in S^{d-1}$ (the unit sphere). Let $\lambda=$ $\lambda(i \xi)$ be the eigenvalues of $(2.14)$, which solve the corresponding characteristic equation

$$
\operatorname{det}\left(\lambda A^{0}+i|\xi| A(\omega)+L\right)=0 .
$$

The stability condition for $(2.2)$ is then formulated as follows. 
Definition 2.5. The system (2.2) satisfies the stability condition at $\bar{u} \in \mathcal{M}$ if the following holds true: Let $\phi \in \mathbb{R}^{m}$ satisfy $\phi \in \mathcal{M}$ (i.e., $L \phi=0$ ) and $\lambda A^{0} \phi+A(\omega) \phi=0$ for a certain real number $\lambda$ and $\omega \in S^{d-1}$. Then $\phi=0$.

This stability condition was first formulated in [12] for a general class of symmetric hyperbolic-parabolic systems including our symmetric hyperbolic systems (2.2) and (2.9). The following characterization of the stability condition was also given in [12].

Theorem 2.6. The following four conditions are equivalent to each other.

- The system (2.2) satisfies the stability condition at $\bar{u} \in \mathcal{M}$.

- $\operatorname{Re} \lambda(i \xi)<0$ for $\xi \neq 0$.

- There is a positive constant c such that $\operatorname{Re} \lambda(i \xi) \leq-\frac{c|\xi|^{2}}{1+|\xi|^{2}}$ for $\xi \in \mathbb{R}^{d}$.

- There is an $m \times m$ matrix $K(\omega)$ depending smoothly on $\omega \in S^{d-1}$ with the following properties:

(i) $K(-\omega)=K(\omega)$ for $\omega \in S^{d-1}$.

(ii) $K(\omega) A^{0}$ is skew-symmetric for $\omega \in S^{d-1}$.

(iii) $[K(\omega) A(\omega)]^{\prime}+L$ is positive definite for $\omega \in S^{d-1}$, where $[X]^{\prime}$ denotes the symmetric part of the matrix $X$, i.e., $[X]^{\prime}=\frac{1}{2}\left(X+X^{T}\right)$.

Remark 2.7. We can also formulate the stability condition for (2.9) at the constant state $\bar{v} \in \mathcal{M}$, corresponding to $\bar{u} \in \mathcal{M}$. It turns out that the stability condition for (2.2) at $\bar{u} \in \mathcal{M}$ is equivalent to the stability condition for (2.9) at $\bar{v} \in \mathcal{M}$.

\section{Energy estimates and global existence}

For completeness, in this section we prove the existence of small-amplitude global smooth solutions to (1.1) admitting an entropy and satisfying the stability condition formulated in [12].

As shown in the previous section, (1.1) is a symmetrizable hyperbolic system for it admits an entropy. According to the general local-in-time existence theory [2], its initial value problem with the initial data $w_{0}(x)$ satisfying $w_{0}-\bar{w} \in H^{s}$ with $s>\frac{d}{2}+1$ has a unique solution $w(x, t)$ satisfying $w-\bar{w} \in C\left(\left[0, T_{0}\right] ; H^{s}\right)$ with $T_{0}$ a positive constant, where $\bar{w} \in \mathcal{E}$ is a fixed constant state. Define $v=v(x, t)$ by (2.8). Then $v(x, t)$ is a unique solution with $v-\bar{v} \in C\left(\left[0, T_{0}\right] ; H^{s}\right)$ to the symmetric dissipative system (2.9) of the normal form, where $\bar{v} \in \mathcal{M}$ is the constant state corresponding to $\bar{w}$. 
The aim of this section is to derive a uniform a priori estimate of solutions $v$ by the energy method. To this end, we introduce

$$
\begin{aligned}
E_{0}(t) & :=\sup _{0 \leq \tau \leq t}\|v(\tau)-\bar{v}\|_{H^{s}} \\
D_{0}(t)^{2} & :=\int_{0}^{t}\left(\|(I-P) v(\tau)\|_{H^{s}}^{2}+\left\|\partial_{x} v(\tau)\right\|_{H^{s-1}}^{2}\right) d \tau \\
M_{0}(t) & :=\sup _{0 \leq \tau \leq t}\|v(\tau)-\bar{v}\|_{L^{\infty}} \\
M_{1}(t) & :=\sup _{0 \leq \tau \leq t}\left\|\partial_{x} v(\tau)\right\|_{L^{\infty}} .
\end{aligned}
$$

Our a priori estimate reads as follows.

Proposition 3.1. Suppose (1.1) admits an entropy and the corresponding symmetric dissipative system (2.9) satisfies the stability condition at $\bar{v} \in \mathcal{M}$. Let $d \geq 1, s>\frac{d}{2}+1$ be an integer, $T>0$, and let $v=v(x, t)$ be a solution with $v-\bar{v} \in C\left([0, T] ; H^{s}\right)$ to the initial value problem of $(2.9)$. Then there is a positive constant $\delta_{0}$ (independent of $T$ ) such that if $E_{0}(T) \leq \delta_{0}$, then the following uniform estimate holds true for $t \in[0, T]$ :

$$
E_{0}(t)^{2}+D_{0}(t)^{2} \leq C\left\|v_{0}-\bar{v}\right\|_{H^{s}}^{2}
$$

where $v_{0}(x):=v(x, 0)$.

Proof. The proof is divided into four steps. Thanks to $s>\frac{d}{2}+1$, it follows from the Sobolev inequality that

$$
M_{0}(t)+M_{1}(t) \leq C E_{0}(t) .
$$

Thus, we always assume that $M_{0}(T)$ is suitably small (independent of $T$ ) and therefore $v=v(x, t)$ takes values in a neighborhood of $\bar{v} \in \mathcal{M}$.

Step 1. First we show the following basic energy estimate

$$
\|v(t)-\bar{v}\|_{L^{2}}^{2}+\int_{0}^{t}\|(I-P) v(\tau)\|_{L^{2}}^{2} \leq C\left\|v_{0}-\bar{v}\right\|_{L^{2}}^{2} .
$$

Let $\bar{w} \in \mathcal{E}$ and $\bar{u} \in \mathcal{M}$ be the constant states corresponding to $\bar{v} \in \mathcal{M}$. Introduce the energy form $\mathcal{H}[w]$ by

$$
\mathcal{H}[w]:=\eta(w)-\eta(\bar{w})-\langle\bar{u}, w-\bar{w}\rangle .
$$

Since $\eta(w)$ is strictly convex in $w$ and $u=\left(D_{w} \eta(w)\right)^{T}, \mathcal{H}[w]$ is equivalent to the quadratic function $|w-\bar{w}|^{2}$ and hence to $|v-\bar{v}|^{2}$. On the other hand, we use (2.7) and (1.1) to compute that

$$
\mathcal{H}[w]_{t}+\sum_{j=1}^{d} \mathcal{Q}^{j}[w]_{x_{j}}=\langle u, g(w)\rangle,
$$


where $\mathcal{Q}^{j}[w]=q^{j}(w)-q^{j}(\bar{w})-\left\langle\bar{u}, f^{j}(w)-f^{j}(\bar{w})\right\rangle$. Since $g(w)=h(u) \in \mathcal{M}^{\perp}$, it follows from (2.5) and (2.6) that

$$
\langle u, g(w)\rangle \leq-c|(I-P) u|^{2} \leq-c|(I-P) v|^{2} .
$$

Here we have used the fact that $u \in \mathcal{M}$ is equivalent to $v \in \mathcal{M}$. Now we integrate (3.4) over $\mathbb{R}^{d} \times[0, t]$ and use (3.5) to obtain the desired estimate (3.3).

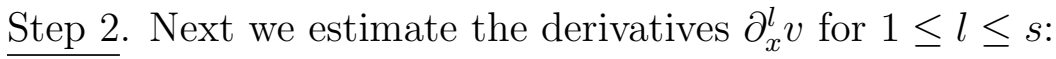

$$
\begin{aligned}
\left\|\partial_{x} v(t)\right\|_{H^{s-1}}^{2} & +\int_{0}^{t}\left\|(I-P) \partial_{x} v(\tau)\right\|_{H^{s-1}}^{2} d \tau \\
& \leq C\left\|\partial_{x} v_{0}\right\|_{H^{s-1}}^{2}+C\left(M_{0}(t)+M_{1}(t)\right) D_{0}(t)^{2}
\end{aligned}
$$

By using (2.11), we rewrite the normal form (2.9) as

$$
\tilde{A}^{0}(v) v_{t}+\sum_{j=1}^{d} \tilde{A}^{j}(v) v_{x_{j}}+L v=\tilde{r}(v)
$$

Applying $\partial_{x}^{l}(1 \leq l \leq s)$ to this equality, we obtain

$$
\tilde{A}^{0}(v) \partial_{x}^{l} v_{t}+\sum_{j=1}^{d} \tilde{A}^{j}(v) \partial_{x}^{l} v_{x_{j}}+L \partial_{x}^{l} v=\tilde{r}^{l} .
$$

Here $\tilde{r}^{l}=\tilde{r}_{A}^{l}+\tilde{r}_{L}^{l}$ with

$$
\begin{aligned}
& \tilde{r}_{A}^{l}=-\sum_{j=1}^{d} \tilde{A}^{0}(v)\left[\partial_{x}^{l}, \tilde{A}^{0}(v)^{-1} \tilde{A}^{j}(v)\right] v_{x_{j}} \\
& \tilde{r}_{L}^{l}=\tilde{A}^{0}(v)\left\{-\left[\partial_{x}^{l}, \tilde{A}^{0}(v)^{-1}\right] L v+\partial_{x}^{l}\left(\tilde{A}^{0}(v)^{-1} \tilde{r}(v)\right)\right\},
\end{aligned}
$$

and $[$,$] denotes the commutator. Taking the inner product of (3.8) with \partial_{x}^{l} v$, we obtain

$$
\begin{aligned}
\left\langle\tilde{A}^{0}(v) \partial_{x}^{l} v, \partial_{x}^{l} v\right\rangle_{t} & +\sum_{j=1}^{d}\left\langle\tilde{A}^{j}(v) \partial_{x}^{l} v, \partial_{x}^{l} v\right\rangle_{x_{j}}+2\left\langle L \partial_{x}^{l} v, \partial_{x}^{l} v\right\rangle \\
& =2\left\langle\tilde{r}^{l}, \partial_{x}^{l} v\right\rangle+\left\langle\left(\tilde{A}^{0}(v)_{t}+\sum_{j=1}^{d} \tilde{A}^{j}(v)_{x_{j}}\right) \partial_{x}^{l} v, \partial_{x}^{l} v\right\rangle .
\end{aligned}
$$

Then the integration over $\mathbb{R}^{d} \times[0, t]$ yields

$$
\left\|\partial_{x}^{l} v(t)\right\|_{L^{2}}^{2}+\int_{0}^{t}\left\|(I-P) \partial_{x}^{l} v(\tau)\right\|_{L^{2}}^{2} d \tau \leq C\left\|\partial_{x}^{l} v_{0}\right\|_{L^{2}}^{2}+C \int_{0}^{t} R^{(l)}(\tau) d \tau,
$$


where $1 \leq l \leq s$ and

$$
R^{(l)}=\int_{\mathbb{R}^{d}}\left\{\left(\left|v_{t}\right|+\left|\partial_{x} v\right|\right)\left|\partial_{x}^{l} v\right|^{2}+\left|\tilde{r}_{A}^{l} \| \partial_{x}^{l} v\right|+\left|\tilde{r}_{L}^{l}\right|\left|(I-P) \partial_{x}^{l} v\right|\right\} d x .
$$

Here we have used the fact that $\tilde{r}_{L}^{l} \in \mathcal{M}^{\perp}$. We claim that

$$
\begin{aligned}
R^{(l)} \leq & C\left(\left\|\partial_{x} v\right\|_{L^{\infty}}+\|(I-P) v\|_{L^{\infty}}\right)\left\|\partial_{x}^{l} v\right\|_{L^{2}}^{2} \\
& +C\|v-\bar{v}\|_{L^{\infty}}\left\|(I-P) \partial_{x}^{l} v\right\|_{L^{2}}^{2}
\end{aligned}
$$

for $1 \leq l \leq s$. In fact, since $\left|v_{t}\right| \leq C\left(\left|\partial_{x} v\right|+|(I-P) v|\right)$ due to (2.9), we have

$$
\int_{\mathbb{R}^{d}}\left(\left|v_{t}\right|+\left|\partial_{x} v\right|\right)\left|\partial_{x}^{l} v\right|^{2} d x \leq C\left(\left\|\partial_{x} v\right\|_{L^{\infty}}+\|(I-P) v\|_{L^{\infty}}\right)\left\|\partial_{x}^{l} v\right\|_{L^{2}}^{2} .
$$

Moreover, by using (3.9) and (2.12), we deduce from the technical calculus inequalities in Sobolev spaces (see $[4,5,10])$ that

$$
\begin{aligned}
& \left\|\tilde{r}_{A}^{l}\right\|_{L^{2}} \leq C\left\|\partial_{x} v\right\|_{L^{\infty}}\left\|\partial_{x}^{l} v\right\|_{L^{2}} \\
& \left\|\tilde{r}_{L}^{l}\right\|_{L^{2}} \leq C\left(\|v-\bar{v}\|_{L^{\infty}}\left\|(I-P) \partial_{x}^{l} v\right\|_{L^{2}}+\|(I-P) v\|_{L^{\infty}}\left\|\partial_{x}^{l} v\right\|_{L^{2}}\right)
\end{aligned}
$$

for $1 \leq l \leq s$. These computations prove the desired estimate (3.11). Now it follows from (3.11) that

$$
\int_{0}^{t} R^{(l)}(\tau) d \tau \leq C\left(M_{0}(t)+M_{1}(t)\right) D_{0}(t)^{2}
$$

for $1 \leq l \leq s$. Thus, summing up (3.10) for $l$ with $1 \leq l \leq s$ gives the desired estimate (3.6).

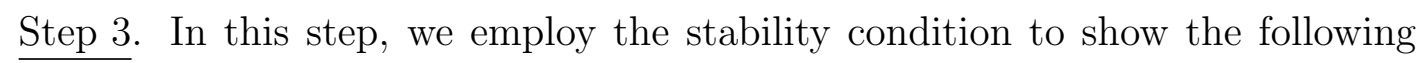
estimate

$$
\begin{aligned}
\int_{0}^{t}\left\|\partial_{x} v(\tau)\right\|_{H^{s-1}}^{2} d \tau \leq & C\left\|v_{0}-\bar{v}\right\|_{H^{s}}^{2}+C M_{0}(t) D_{0}(t)^{2} \\
& +C\left(\|v(t)-\bar{v}\|_{H^{s}}^{2}+\int_{0}^{t}\|(I-P) v(\tau)\|_{H^{s}}^{2} d \tau\right)
\end{aligned}
$$

For this purpose, we set $z=v-\bar{v}$ and rewrite (2.9) as

$$
\tilde{A}^{0} z_{t}+\sum_{j=1}^{d} \tilde{A}^{j} z_{x_{j}}+L z=b,
$$

where $\tilde{A}^{0}=\tilde{A}^{0}(\bar{v}), \tilde{A}^{j}=\tilde{A}^{j}(\bar{v})$ and $L$ are constant matrices, and $b=b_{A}+b_{L}$ with

$$
\begin{aligned}
& b_{A}=-\sum_{j=1}^{d} \tilde{A}^{0}\left(\tilde{A}^{0}(v)^{-1} \tilde{A}^{j}(v)-\left(\tilde{A}^{0}\right)^{-1} \tilde{A}^{j}\right) v_{x_{j}} \\
& b_{L}=\tilde{A}^{0}\left\{-\left(\tilde{A}^{0}(v)^{-1}-\left(\tilde{A}^{0}\right)^{-1}\right) L v+\tilde{A}^{0}(v)^{-1} \tilde{r}(v)\right\} .
\end{aligned}
$$


Taking the Fourier transform of (3.13), we obtain

$$
\tilde{A}^{0} \hat{z}_{t}+i|\xi| \tilde{A}(\omega) \hat{z}+L \hat{z}=\hat{b}
$$

with $\tilde{A}(\omega)=\sum_{j=1}^{d} \tilde{A}^{j} \omega_{j}$ and $\omega=\frac{\xi}{|\xi|}$. Recall the matrix $\tilde{K}(\omega)$ in Theorem 2.6 (Remark 2.1). We multiply (3.15) with $-i|\xi| \tilde{K}(\omega)$ and then take the inner product with $\hat{z}$. From the real part of the resulting equality we have

$$
\begin{aligned}
& -\frac{1}{2}|\xi|\left(i \tilde{K}(\omega) \tilde{A}^{0} \hat{z}, \hat{z}\right)_{t}+|\xi|^{2}\left([\tilde{K}(\omega) \tilde{A}(\omega)]^{\prime} \hat{z}, \hat{z}\right) \\
& =\operatorname{Re}\{i|\xi|(\tilde{K}(\omega) L \hat{z}, \hat{z})\}-\operatorname{Re}\{i|\xi|(\tilde{K}(\omega) \hat{b}, \hat{z})\}
\end{aligned}
$$

Here we have used the fact that $i \tilde{K}(\omega) \tilde{A}^{0}$ is hermitian. Since $[\tilde{K}(\omega) \tilde{A}(\omega)]^{\prime}+L$ is positive definite, we have

$$
\left([\tilde{K}(\omega) \tilde{A}(\omega)]^{\prime} \hat{z}, \hat{z}\right) \geq c|\hat{z}|^{2}-C|(I-P) \hat{z}|^{2} .
$$

Moreover, for any $\epsilon>0$, we have

$$
|\operatorname{Re}\{i|\xi|(\tilde{K}(\omega) L \hat{z}, \hat{z})\}| \leq \epsilon|\xi|^{2}|\hat{z}|^{2}+\epsilon^{-1} C|(I-P) \hat{z}|^{2} .
$$

Substituting these two inequalities into (3.16) and taking $\epsilon>0$ suitably small, we obtain

$$
-|\xi|\left(i \tilde{K}(\omega) \tilde{A}^{0} \hat{z}, \hat{z}\right)_{t}+c|\xi|^{2}|\hat{z}|^{2} \leq C\left(1+|\xi|^{2}\right)|(I-P) \hat{z}|^{2}+C|\xi||\hat{b}||\hat{z}| .
$$

Now, multiplying this inequality by $|\xi|^{2 l}(0 \leq l \leq s-1)$ and integrating over $\mathbb{R}_{\xi}^{d} \times[0, t]$ gives

$$
\begin{aligned}
\int_{0}^{t}\left\|\partial_{x}^{l+1} v(\tau)\right\|_{L^{2}}^{2} d \tau \leq & C\left\|\partial_{x}^{l}\left(v_{0}-\bar{v}\right)\right\|_{H^{1}}^{2}+C\left(\left\|\partial_{x}^{l}(v(t)-\bar{v})\right\|_{H^{1}}^{2}\right. \\
& \left.+\int_{0}^{t}\left\|(I-P) \partial_{x}^{l} v(\tau)\right\|_{H^{1}}^{2} d \tau\right)+C \int_{0}^{t} S^{(l)}(\tau) d \tau
\end{aligned}
$$

where $0 \leq l \leq s-1$ and $S^{(l)}=\int_{\mathbb{R}^{d}}\left(\left|\partial_{x}^{l} b_{A}\right|+\left|\partial_{x}^{l} b_{L}\right|\right)\left|\partial_{x}^{l+1} v\right| d x$. On the other hand, with the expressions of $b_{A}$ and $b_{L}$ in (3.14), we deduce from the calculus inequalities in Sobolev spaces that

$$
\begin{aligned}
\left\|\partial_{x}^{l} b_{A}\right\|_{L^{2}} & \leq C\|v-\bar{v}\|_{L^{\infty}}\left\|\partial_{x}^{l+1} v\right\|_{L^{2}} \\
\left\|\partial_{x}^{l} b_{L}\right\|_{L^{2}} & \leq C\left(\|v-\bar{v}\|_{L^{\infty}}\left\|(I-P) \partial_{x}^{l} v\right\|_{L^{2}}+\|(I-P) v\|_{L^{\infty}}\left\|\partial_{x}^{l}(v-\bar{v})\right\|_{L^{2}}\right)
\end{aligned}
$$

for $0 \leq l \leq s-1$, where the last term $\|(I-P) v\|_{L^{\infty}}\left\|\partial_{x}^{l}(v-\bar{v})\right\|_{L^{2}}$ is absent for $l=0$. From these estimates it follows that

$$
\begin{aligned}
S^{(l)} \leq & C\|v-\bar{v}\|_{L^{\infty}}\left(\left\|(I-P) \partial_{x}^{l} v\right\|_{L^{2}}^{2}+\left\|\partial_{x}^{l+1} v\right\|_{L^{2}}^{2}\right) \\
& +C\|(I-P) v\|_{L^{\infty}}\left\|\partial_{x}^{l}(v-\bar{v})\right\|_{L^{2}}\left\|\partial_{x}^{l+1} v\right\|_{L^{2}}
\end{aligned}
$$


for $0 \leq l \leq s-1$, where the last term on the right-hand side is absent for $l=0$. Consequently, we have

$$
\int_{0}^{t} S^{(l)}(\tau) d \tau \leq C M_{0}(t) D_{0}(t)^{2}
$$

for $0 \leq l \leq s-1$. Thus, adding (3.18) for $l$ with $0 \leq l \leq s-1$ yields the desired estimate (3.12).

Step 4. Finally, we combine (3.3), (3.6) and (3.12) to get

$$
E_{0}(t)^{2}+D_{0}(t)^{2} \leq C\left\|v_{0}-\bar{v}\right\|_{H^{s}}^{2}+C\left(M_{0}(t)+M_{1}(t)\right) D_{0}(t)^{2} .
$$

Assume that $E_{0}(T)$ is suitably small. We use (3.2) to conclude the desired $a$ priori estimate (3.1). This completes the proof of Proposition 3.1.

On the basis of the a priori estimate (3.1), we use the standard continuation argument to obtain the following global existence theorem - a refinement of that in $[9]$.

Theorem 3.2. Suppose (1.1) possesses an entropy and the corresponding symmetric dissipative system (2.9) satisfies the stability condition at $\bar{v} \in \mathcal{M}$, where $\bar{v}$ is the constant state corresponding to a given constant state $\bar{w} \in \mathcal{E}$. Let $d \geq 1$ and let the initial data $w_{0}(x)$ satisfy $w_{0}-\bar{w} \in H^{s}$ with $s>\frac{d}{2}+1$. Then there is a positive constant $\delta_{1}$ such that if $\left\|w_{0}-\bar{w}\right\|_{H^{s}} \leq \delta_{1}$, the initial value problem of (1.1) has a unique global solution $w$ with $w-\bar{w} \in C\left([0, \infty) ; H^{s}\right)$, which satisfies the following uniform estimate:

$$
\|w(t)-\bar{w}\|_{H^{s}}^{2}+\int_{0}^{t}\left(\|(I-P) v(\tau)\|_{H^{s}}^{2}+\left\|\partial_{x} w(\tau)\right\|_{H^{s-1}}^{2}\right) d \tau \leq C\left\|w_{0}-\bar{w}\right\|_{H^{s}}^{2}
$$

for $t \geq 0$. Here $v$ is defined in (2.8) and $P$ is the orthogonal projection onto $\mathcal{M}$. Moreover, the solution $w$ converges to the constant state $\bar{w}$ as $t$ tends to infinity, namely,

$$
\left\|\partial_{x}^{l}(w(t)-\bar{w})\right\|_{L^{\infty}} \longrightarrow 0
$$

as $t \rightarrow \infty$, where $0 \leq l \leq s-s_{0}$ with $s_{0}=\left[\frac{d}{2}\right]+1$.

Remark 3.3. It is clear that the global solution $v$ to (2.9) satisfies

$$
\|v(t)-\bar{v}\|_{H^{s}}^{2}+\int_{0}^{t}\left(\|(I-P) v(\tau)\|_{H^{s}}^{2}+\left\|\partial_{x} v(\tau)\right\|_{H^{s-1}}^{2}\right) d \tau \leq C\left\|v_{0}-\bar{v}\right\|_{H^{s}}^{2}
$$

for $t \geq 0$, where $v_{0}(x)$ is the corresponding initial data. 


\section{Time-weighted energy estimates}

This section is devoted to time-weighted energy estimates for the global solution $v$, constructed in the previous section, to the initial value problem of (2.9). To this end, we introduce the following time-weighted quantities:

$$
\begin{aligned}
E(t)^{2}:= & \sum_{k=0}^{s} \sup _{0 \leq \tau \leq t}(1+\tau)^{k}\left\|\partial_{x}^{k}(v(\tau)-\bar{v})\right\|_{H^{s-k}}^{2} \\
D(t)^{2}:= & \sum_{k=0}^{s} \int_{0}^{t}(1+\tau)^{k}\left\|(I-P) \partial_{x}^{k} v(\tau)\right\|_{H^{s-k}}^{2} d \tau \\
& +\sum_{k=0}^{s-1} \int_{0}^{t}(1+\tau)^{k}\left\|\partial_{x}^{k+1} v(\tau)\right\|_{H^{s-k-1}}^{2} d \tau \\
M(t):= & \sup _{0 \leq \tau \leq t}(1+\tau)\left(\left\|\partial_{x} v(\tau)\right\|_{L^{\infty}}+\|(I-P) v(\tau)\|_{L^{\infty}}\right) .
\end{aligned}
$$

About these quantities, we have

Proposition 4.1. Under the conditions of Theorem 3.2, we have

$$
E(t)^{2}+D(t)^{2} \leq C\left\|v_{0}-\bar{v}\right\|_{H^{s}}^{2}+C M(t) D(t)^{2} .
$$

Proof. This proof consists of three steps. As in the proof of Proposition 3.1, we always assume that $M_{0}(t)$ is suitably small so that $v=v(x, t)$ takes values in a neighborhood of $\bar{v} \in \mathcal{M}$.

Step 1: We first show that

$$
\begin{aligned}
& (1+t)^{k}\left\|\partial_{x}^{k} v(t)\right\|_{H^{s-k}}^{2}+\int_{0}^{t}(1+\tau)^{k}\left\|(I-P) \partial_{x}^{k} v(\tau)\right\|_{H^{s-k}}^{2} d \tau \\
& \leq C\left\|\partial_{x}^{k} v_{0}\right\|_{H^{s-k}}^{2}+C \int_{0}^{t}(1+\tau)^{k-1}\left\|\partial_{x}^{k} v(\tau)\right\|_{H^{s-k}}^{2} d \tau \\
& \quad+C\left(M_{0}(t)+M(t)\right) D(t)^{2}
\end{aligned}
$$

for $1 \leq k \leq s$. To do this, we take the inner product of (3.8) with $\partial_{x}^{l} v$ and integrate over $\mathbb{R}^{d}$. Then we multiply the resulting equation by $(1+t)^{k}(1 \leq$ $k \leq s)$ and integrate over $[0, t]$. This yields as a counterpart of $(3.10)$ that

$$
\begin{aligned}
& (1+t)^{k}\left\|\partial_{x}^{l} v(t)\right\|_{L^{2}}^{2}+\int_{0}^{t}(1+\tau)^{k}\left\|(I-P) \partial_{x}^{l} v(\tau)\right\|_{L^{2}}^{2} d \tau \\
& \leq C\left\|\partial_{x}^{l} v_{0}\right\|_{L^{2}}^{2}+C \int_{0}^{t}(1+\tau)^{k-1}\left\|\partial_{x}^{l} v(\tau)\right\|_{L^{2}}^{2} d \tau+C \int_{0}^{t}(1+\tau)^{k} R^{(l)}(\tau) d \tau
\end{aligned}
$$


where $1 \leq k, l \leq s$. Moreover, we see from (3.11) that $\int_{0}^{t}(1+\tau)^{k} R^{(l)}(\tau) d \tau \leq$ $C\left(M_{0}(t)+M(t)\right) D(t)^{2}$ for $1 \leq k \leq l \leq s$. Thus, adding (4.4) for $l$ with $k \leq l \leq s$, we arrive at the desired estimate (4.3).

Step 2: In this step, we show that

$$
\begin{aligned}
& \int_{0}^{t}(1+\tau)^{k}\left\|\partial_{x}^{k+1} v(\tau)\right\|_{H^{s-k-1}}^{2} d \tau \\
& \leq C\left\|\partial_{x}^{k} v_{0}\right\|_{H^{s-k}}^{2}+C(1+t)^{k}\left\|\partial_{x}^{k} v(t)\right\|_{H^{s-k}}^{2} \\
& \quad+C \int_{0}^{t}(1+\tau)^{k}\left\|(I-P) \partial_{x}^{k} v(\tau)\right\|_{H^{s-k}}^{2} d \tau \\
& \quad+C \int_{0}^{t}(1+\tau)^{k-1}\left\|\partial_{x}^{k} v(\tau)\right\|_{H^{s-k}}^{2} d \tau+C\left(M_{0}(t)+M(t)\right) D(t)^{2}
\end{aligned}
$$

for $1 \leq k \leq s-1$. For this purpose, we multiply (3.17) with $|\xi|^{2 l}(1 \leq l \leq s-1)$ and integrate over $\mathbb{R}_{\xi}^{d}$. Then we multiply the resulting equation by $(1+t)^{k}$ $(1 \leq k \leq s-1)$ and integrate over $[0, t]$. This yields

$$
\begin{aligned}
& \int_{0}^{t}(1+\tau)^{k}\left\|\partial_{x}^{l+1} v(\tau)\right\|_{L^{2}}^{2} d \tau \\
& \leq C\left\|\partial_{x}^{l} v_{0}\right\|_{H^{1}}^{2}+C(1+t)^{k}\left\|\partial_{x}^{l} v(t)\right\|_{H^{1}}^{2} \\
& \quad+C \int_{0}^{t}(1+\tau)^{k}\left\|(I-P) \partial_{x}^{l} v(\tau)\right\|_{H^{1}}^{2} d \tau \\
& \quad+C \int_{0}^{t}(1+\tau)^{k-1}\left\|\partial_{x}^{l} v(\tau)\right\|_{H^{1}}^{2} d \tau+C \int_{0}^{t}(1+\tau)^{k} S^{(l)}(\tau) d \tau
\end{aligned}
$$

where $1 \leq k, l \leq s-1$. Moreover, we deduce from (3.19) that $\int_{0}^{t}(1+\tau)^{k} S^{(l)}(\tau) d \tau$ $\leq C\left(M_{0}(t)+M(t)\right) D(t)^{2}$ for $k \leq l \leq s-1$. Thus, adding (4.6) for $l$ with $k \leq l \leq s-1$, we arrive at the desired estimate (4.5).

Step 3: Having estimates (4.3) and (4.5), we can easily deduce that

$$
\begin{aligned}
& (1+t)^{k}\left\|\partial_{x}^{k}(v(t)-\bar{v})\right\|_{H^{s-k}}^{2}+\int_{0}^{t}(1+\tau)^{k}\left\|(I-P) \partial_{x}^{k} v(\tau)\right\|_{H^{s-k}}^{2} d \tau \\
& \leq C\left\|v_{0}-\bar{v}\right\|_{H^{s}}^{2}+C\left(M_{0}(t)+M(t)\right) D(t)^{2}
\end{aligned}
$$

for $0 \leq k \leq s$ and

$$
\int_{0}^{t}(1+\tau)^{k}\left\|\partial_{x}^{k+1} v(\tau)\right\|_{H^{s-k-1}}^{2} d \tau \leq C\left\|v_{0}-\bar{v}\right\|_{H^{s}}^{2}+C\left(M_{0}(t)+M(t)\right) D(t)^{2}
$$

for $0 \leq k \leq s-1$. Note that these two inequalities, together with the smallness of $M_{0}(t)$, simply give the desired time-weighted estimate (4.2). 
We prove (4.7) and (4.8) by induction on $k$. First we observe that for $k=0$, (4.7) and (4.8) simply follow from (3.20). Next, let $1 \leq k \leq s$ and assume that (4.8) holds true for $k-1$. Then it follows from (4.3) that (4.7) holds for $k$. Furthermore, substituting (4.7) with $k$ and (4.8) with $k-1$ into (4.5), we find that (4.8) holds true for $k$. This proves Proposition 4.1.

Next we introduce

$$
E_{\perp}(t):=\sum_{k=0}^{s-1} \sup _{0 \leq \tau \leq t}(1+\tau)^{\frac{1}{2}(k+1)}\left\|(I-P) \partial_{x}^{k} v(\tau)\right\|_{L^{2}}
$$

About this time-weighted quantity, we have

Proposition 4.2. Under the conditions of Proposition 4.1, it holds that

$$
E_{\perp}(t) \leq C\left\|v_{0}-\bar{v}\right\|_{H^{s-1}}+C E(t)+C M(t) E(t) .
$$

Proof. Let $\left(v_{1}, v_{2}\right)$ be the partition of $v$ according to the orthogonal decomposition $\mathbb{R}^{m}=\mathcal{M} \oplus \mathcal{M}^{\perp}$. From Theorem 2.4 and (3.7) we see that

$$
\tilde{A}_{22}^{0}(v) v_{2 t}+\sum_{j=1}^{d}\left(\tilde{A}_{21}^{j}(v) v_{1 x_{j}}+\tilde{A}_{22}^{j}(v) v_{2 x_{j}}\right)+L_{22} v_{2}=\tilde{r}_{2}(v) .
$$

Here $\tilde{A}_{22}^{0}(v)$ and $L_{22}$ are real symmetric and positive definite. We rewrite this equation as

$$
\tilde{A}_{22}^{0} v_{2 t}+L_{22} v_{2}=\tilde{b}_{2}
$$

where $\tilde{A}_{22}^{0}=\tilde{A}_{22}^{0}(\bar{v})$ and

$$
\begin{aligned}
\tilde{b}_{2}= & \tilde{A}_{22}^{0}\left\{-\tilde{A}_{22}^{0}(v)^{-1} \sum_{j=1}^{d}\left(\tilde{A}_{21}^{j}(v) v_{1 x_{j}}+\tilde{A}_{22}^{j}(v) v_{2 x_{j}}\right)\right. \\
& \left.-\left(\tilde{A}_{22}^{0}(v)^{-1}-\left(\tilde{A}_{22}^{0}\right)^{-1}\right) L_{22} v_{2}+\tilde{A}_{22}^{0}(v)^{-1} \tilde{r}_{2}(v)\right\} .
\end{aligned}
$$

Note that $\left(\tilde{A}_{22}^{0}\right)^{-1} L_{22}$ is a positive definite matrix. Solving $v_{2}$ from the ordinary differential equation (4.11), applying $\partial_{x}^{k}$ with $0 \leq k \leq s-1$ and then taking the $L^{2}$-norm, we get

$$
\left\|(I-P) \partial_{x}^{k} v(t)\right\|_{L^{2}} \leq C e^{-c t}\left\|(I-P) \partial_{x}^{k} v_{0}\right\|_{L^{2}}+C \int_{0}^{t} e^{-c(t-\tau)}\left\|\partial_{x}^{k} \tilde{b}_{2}(\tau)\right\|_{L^{2}} .
$$

Now we use the calculus inequalities in Sobolev spaces to obtain

$$
\begin{aligned}
\left\|\partial_{x}^{k} \tilde{b}_{2}\right\|_{L^{2}} \leq & C\left\|\partial_{x}^{k+1} v\right\|_{L^{2}}+C\left(\|v-\bar{v}\|_{L^{\infty}}\left\|(I-P) \partial_{x}^{k} v\right\|_{L^{2}}\right. \\
& \left.+\|(I-P) v\|_{L^{\infty}}\left\|\partial_{x}^{k}(v-\bar{v})\right\|_{L^{2}}\right)
\end{aligned}
$$


for $0 \leq k \leq s-1$, where the last term on the right-hand side is absent for $k=0$. Thus, it follows from (4.1) and (4.9) that

$$
\begin{aligned}
& \int_{0}^{t} e^{-c(t-\tau)}\left\|\partial_{x}^{k} \tilde{b}_{2}(\tau)\right\|_{L^{2}} d \tau \\
& \leq C\left(E(t)+M_{0}(t) E_{\perp}(t)+M(t) E(t)\right) \int_{0}^{t} e^{-c(t-\tau)}(1+\tau)^{-\frac{1}{2}(k+1)} d \tau \\
& \leq C\left(E(t)+M_{0}(t) E_{\perp}(t)+M(t) E(t)\right)(1+t)^{-\frac{1}{2}(k+1)} .
\end{aligned}
$$

Substituting this into (4.12), we arrive at

$$
\begin{aligned}
& (1+t)^{\frac{1}{2}(k+1)}\left\|(I-P) \partial_{x}^{k} v(t)\right\|_{L^{2}} \\
& \leq C\left\|v_{0}-\bar{v}\right\|_{H^{s-1}}+C\left(E(t)+M_{0}(t) E_{\perp}(t)+M(t) E(t)\right)
\end{aligned}
$$

for $0 \leq k \leq s-1$. Summing up the last inequality for $k$ and using the smallness of $M_{0}(t)$, we arrive at the desired estimate (4.10). This completes the proof.

From the above two propositions, we can derive suitable decay estimates for the solution $v$ without assuming the condition $v_{0}-\bar{v} \in L^{1}$, provided that $d \geq 2$. The decay rate obtained is optimal in the situation where $v_{0}-\bar{v} \in H^{s}$ and the result reads as follows.

Theorem 4.3. Let $d \geq 2$ and $s>\frac{d}{2}+1$. Under the conditions of Theorem 3.2, suppose that $\left\|v_{0}-\bar{v}\right\|_{H^{s}}$ is sufficiently small. Then the following decay estimates hold:

$$
\left\|\partial_{x}^{k}(v(t)-\bar{v})\right\|_{L^{p}} \leq C\left\|v_{0}-\bar{v}\right\|_{H^{s}}(1+t)^{-\gamma_{p}^{\prime}-\frac{k}{2}}
$$

for $p \in[2, \infty]$ and $0 \leq k \leq s-2 \gamma_{p}^{\prime}\left(k \neq s-\frac{d}{2}\right.$ if $\left.p=\infty\right)$, and

$$
\left\|(I-P) \partial_{x}^{k} v(t)\right\|_{L^{p}} \leq C\left\|v_{0}-\bar{v}\right\|_{H^{s}}(1+t)^{-\gamma_{p}^{\prime}-\frac{1}{2}(k+1)}
$$

for $p \in[2, \infty]$ and $0 \leq k \leq s-1-2 \gamma_{p}^{\prime}\left(k \neq s-1-\frac{d}{2}\right.$ if $\left.p=\infty\right)$, where $\gamma_{p}^{\prime}=\frac{d}{2}\left(\frac{1}{2}-\frac{1}{p}\right)$ is the decay exponent of the $L^{p}-L^{2}$ decay estimate for the linear heat equation.

Proof. Recall the Gagliardo-Nirenberg inequality

$$
\|f\|_{L^{\infty}} \leq C\left\|\partial_{x}^{s_{0}} f\right\|_{L^{2}}^{\theta}\|f\|_{L^{2}}^{1-\theta}
$$

with $s_{0}=\left[\frac{d}{2}\right]+1$ and $\theta=\frac{d}{2 s_{0}}$. We deduce that

$$
\begin{aligned}
\left\|\partial_{x} v(t)\right\|_{L^{\infty}} & \leq C E(t)(1+t)^{-\frac{d}{4}-\frac{1}{2}} \\
\|(I-P) v(t)\|_{L^{\infty}} & \leq C E_{\perp}(t)(1+t)^{-\frac{d}{4}-\frac{1}{2}} .
\end{aligned}
$$


Thus, for $d \geq 2$, we have

$$
M(t) \leq C\left(E(t)+E_{\perp}(t)\right) .
$$

Combining this with (4.2) and (4.10), we conclude that $E(t)+E_{\perp}(t)+D(t) \leq$ $C\left\|v_{0}-\bar{v}\right\|_{H^{s}}$, provided that $\left\|v_{0}-\bar{v}\right\|_{H^{s}}$ is sufficiently small. In particular, we have

$$
\left\|\partial_{x}^{k}(v(t)-\bar{v})\right\|_{L^{2}} \leq C\left\|v_{0}-\bar{v}\right\|_{H^{s}}(1+t)^{-\frac{k}{2}}
$$

for $0 \leq k \leq s$, and

$$
\left\|(I-P) \partial_{x}^{k} v(t)\right\|_{L^{2}} \leq C\left\|v_{0}-\bar{v}\right\|_{H^{s}}(1+t)^{-\frac{1}{2}(k+1)}
$$

for $0 \leq k \leq s-1$. Now the desired estimates follow from the GagliardoNirenberg inequality

$$
\left\|\partial_{x}^{k} f\right\|_{L^{p}} \leq C\left\|\partial_{x}^{m} f\right\|_{L^{2}}^{\theta}\|f\|_{L^{2}}^{1-\theta}
$$

with $0 \leq k \leq m, p \in[2, \infty]$ and $\theta=\frac{k+2 \gamma_{p}^{\prime}}{m} \leq 1(<1$ if $p=\infty)$. This completes the proof.

\section{Decay estimates for linear problems}

In this section, we consider the following linearization of the nonlinear system (1.1):

$$
A^{0} z_{t}+\sum_{j=1}^{d} A^{j} z_{x_{j}}+L z=\sum_{j=1}^{d} p_{x_{j}}^{j}+q .
$$

See (6.1) for the derivation of this linearization. In (5.1), $A^{0}=A^{0}(\bar{u}), A^{j}=$ $A^{j}(\bar{u}), L=L(\bar{u})$ (see $(2.2)$ ) with $\bar{u} \in \mathcal{M}$ the constant state corresponding to $\bar{w} \in \mathcal{E}, p^{j}$ and $q$ are given functions of $(x, t)$ satisfying

$$
q(x, t) \in \mathcal{M}^{\perp} \text { for }(x, t) \in \mathbb{R}^{d} \times[0, \infty) .
$$

First, we apply the energy method in the Fourier space to derive the following pointwise estimate for the Fourier image

$$
\hat{z}(\xi, t):=(2 \pi)^{-\frac{d}{2}} \int_{\mathbb{R}^{d}} e^{-i \xi \cdot x} z(x, t) d x .
$$

Proposition 5.1. Set $\rho(\xi)=\frac{|\xi|^{2}}{1+|\xi|^{2}}$. Under the conditions of Theorem 3.2, the Fourier image of the solution $z=z(x, t)$ to the linear system (5.1) satisfies the following pointwise estimate

$$
\begin{aligned}
|\hat{z}(\xi, t)|^{2} \leq & C e^{-c \rho(\xi) t}\left|\hat{z}_{0}(\xi)\right|^{2} \\
& +C \int_{0}^{t} e^{-c \rho(\xi)(t-\tau)}\left(\left(1+|\xi|^{2}\right)|\hat{p}(\xi, \tau)|^{2}+|\hat{q}(\xi, \tau)|^{2}\right) d \tau,
\end{aligned}
$$

where $z_{0}(x)$ is the initial data and $p=\left(p^{1}, p^{2}, \ldots, p^{d}\right)$ is an $m \times d$ matrix. 
Proof. Taking the Fourier transform of (5.1), we obtain

$$
A^{0} \hat{z}_{t}+i|\xi| A(\omega) \hat{z}+L \hat{z}=i|\xi| \hat{p} \omega+\hat{q}
$$

where $A(\omega)=\sum_{j=1}^{d} A^{j} \omega_{j}$ and $\omega=\frac{\xi}{|\xi|}$. We take the inner product of (5.4) with $\hat{z}$. Since $A^{0}, A(\omega)$ and $L$ are real symmetric, the real part of the resulting equation is

$$
\frac{1}{2}\left(A^{0} \hat{z}, \hat{z}\right)_{t}+(L \hat{z}, \hat{z})=\operatorname{Re}\{i|\xi|(\hat{p} \omega, \hat{z})\}+\operatorname{Re}(\hat{q}, \hat{z}) .
$$

Since $L$ is positive definite on $\mathcal{M}^{\perp}$ and $\hat{q} \in \mathcal{M}^{\perp}$ due to (5.2), there is a positive constant $c_{1}$ such that (5.5) can be estimated as

$$
\frac{1}{2}\left(A^{0} \hat{z}, \hat{z}\right)_{t}+c_{1}|(I-P) \hat{z}|^{2} \leq \epsilon|\xi|^{2}\left(1+|\xi|^{2}\right)^{-1}|\hat{z}|^{2}+\epsilon^{-1} C\left(1+|\xi|^{2}\right)|\hat{p}|^{2}+C|\hat{q}|^{2}
$$

for any $\epsilon>0$.

Next we use the matrix $K=K(\omega)$ in Theorem 2.6. Multiply (5.4) with $-i|\xi| K(\omega)$ and then take the inner product with $\hat{z}$. Since $i K(\omega) A^{0}$ is hermitian, the real part of the resulting equation is

$$
\begin{aligned}
& -\frac{1}{2}|\xi|\left(i K(\omega) A^{0} \hat{z}, \hat{z}\right)_{t}+|\xi|^{2}\left([K(\omega) A(\omega)]^{\prime} \hat{z}, \hat{z}\right) \\
& =\operatorname{Re}\{i|\xi|(K(\omega) L \hat{z}, \hat{z})\}+\operatorname{Re}\left\{|\xi|^{2}(K(\omega) \hat{p} \omega, \hat{z})\right\}-\operatorname{Re}\{i|\xi|(K(\omega) \hat{q}, \hat{z})\} .
\end{aligned}
$$

As $[K(\omega) A(\omega)]^{\prime}+L$ is positive definite, there are positive constants $c_{2}$ and $C_{3}$ such that the above equality can be estimated as

$$
\begin{aligned}
-\frac{1}{2}|\xi|\left(i K(\omega) A^{0} \hat{z}, \hat{z}\right)_{t}+c_{2}|\xi|^{2}|\hat{z}|^{2} \leq & C_{3}\left(1+|\xi|^{2}\right)|(I-P) \hat{z}|^{2} \\
& +C|\xi|^{2}|\hat{p}|^{2}+C|\hat{q}|^{2} .
\end{aligned}
$$

Now let $\kappa$ be a positive constant to be specified later. We multiply (5.7) by $\kappa\left(1+|\xi|^{2}\right)^{-1}$ and add the resulting inequality and (5.6). This yields

$$
\begin{aligned}
& \frac{1}{2}\left(E_{\kappa}(\xi) \hat{z}, \hat{z}\right)_{t}+\left(c_{1}-\kappa C_{3}\right)|(I-P) \hat{z}|^{2}+\left(\kappa c_{2}-\epsilon\right) \rho(\xi)|\hat{z}|^{2} \\
& \leq C(\epsilon, \kappa)\left(1+|\xi|^{2}\right)|\hat{p}|^{2}+C(\kappa)|\hat{q}|^{2}
\end{aligned}
$$

for some constants $C(\epsilon, \kappa)$ and $C(\kappa)$, where $\rho(\xi)=\frac{|\xi|}{1+|\xi|^{2}}$ and

$$
E_{\kappa}(\xi)=A^{0}-\frac{\kappa|\xi|}{1+|\xi|^{2}} i K(\omega) A^{0}
$$

Note that $E_{\kappa}(\xi)$ is hermitian and $A^{0}$ is positive definite. We choose $\kappa>0$ so small that $c_{1}-\kappa C_{3} \geq 0$ and that $E_{\kappa}(\xi)$ is positive definite uniformly in $\xi \in \mathbb{R}_{\xi}^{n}$ :

$$
c|y|^{2} \leq\left(E_{\kappa}(\xi) y, y\right) \leq C|y|^{2}
$$


for any $y \in \mathbb{C}^{m}$. Then we take $\epsilon=\frac{\kappa c_{2}}{2}$. Thus we can regard (5.8) as an ordinary differential inequality for $Z=\left(E_{\kappa}(\xi) \hat{z}, \hat{z}\right)$ :

$$
Z_{t}+c \rho(\xi) Z \leq C\left(1+|\xi|^{2}\right)|\hat{p}|^{2}+C|\hat{q}|^{2} .
$$

Applying the Gronwall inequality and using (5.9) we deduce the desired estimate (5.3). This completes the proof.

As a corollary of Proposition 5.1, we have the following decay estimate for the linear problem (5.1).

Lemma 5.2. Under the conditions of Theorem 3.2, the solution $z=z(x, t)$ to the linear problem (5.1) satisfies the following estimate:

$$
\begin{aligned}
\left\|\partial_{x}^{k} z(t)\right\|_{L^{2}}^{2} \leq & C e^{-c t}\left\|\partial_{x}^{k} z_{0}\right\|_{L^{2}}^{2}+C(1+t)^{-\frac{d}{2}-k}\left\|z_{0}\right\|_{L^{1}}^{2} \\
& +C \int_{0}^{t} e^{-c(t-\tau)}\left(\left\|\partial_{x}^{k} p(\tau)\right\|_{H^{1}}^{2}+\left\|\partial_{x}^{k} q(\tau)\right\|_{L^{2}}^{2}\right) d \tau \\
& +C \int_{0}^{\frac{t}{2}}(1+t-\tau)^{-\frac{d}{2}-k}\left(\|p(\tau)\|_{L^{1}}^{2}+\|q(\tau)\|_{L^{1}}^{2}\right) d \tau \\
& +C \int_{\frac{t}{2}}^{t}(1+t-\tau)^{-\frac{d}{2}}\left(\left\|\partial_{x}^{k} p(\tau)\right\|_{L^{1}}^{2}+\left\|\partial_{x}^{k} q(\tau)\right\|_{L^{1}}^{2}\right) d \tau .
\end{aligned}
$$

for any nonnegative integer $k$.

Proof. Multiplying (5.3) with $|\xi|^{2 k}$ and integrating the resulting inequality over $\mathbb{R}_{\xi}^{d}$, we use the Plancherel theorem to obtain

$$
\begin{aligned}
\left\|\partial_{x}^{k} z(t)\right\|_{L^{2}}^{2} \leq & C \int_{\mathbb{R}_{\xi}^{d}}|\xi|^{2 k} e^{-c \rho(\xi) t}\left|\hat{z}_{0}(\xi)\right|^{2} d \xi \\
& +C \int_{0}^{t} \int_{\mathbb{R}_{\xi}^{d}}|\xi|^{2 k} e^{-c \rho(\xi)(t-\tau)}\left(\left(1+|\xi|^{2}\right)|\hat{p}(\xi, \tau)|^{2}+|\hat{q}(\xi, \tau)|^{2}\right) d \xi d \tau .
\end{aligned}
$$

Thus, the lemma follows from the estimate

$$
\int_{\mathbb{R}_{\xi}^{d}}|\xi|^{2 k} e^{-c \rho(\xi) t}|\hat{h}(\xi)|^{2} d \xi \leq e^{-c t}\left\|\partial_{x}^{k} h\right\|_{L^{2}}^{2}+C(1+t)^{-\frac{d}{2}-(k-l)}\left\|\partial_{x}^{l} h\right\|_{L^{1}}^{2},
$$

where $0 \leq l \leq k$. It remains to prove this inequality. Since $\rho(\xi)=\frac{|\xi|^{2}}{1+|\xi|^{2}} \geq \frac{|\xi|^{2}}{2}$ for $|\xi| \leq 1$, we deduce from the definition of the Fourier transform that

$$
\begin{aligned}
\int_{|\xi| \leq 1}|\xi|^{2 k} e^{-c \rho(\xi) t}|\hat{h}(\xi)|^{2} d \xi & \leq \sup _{|\xi| \leq 1}\left(|\xi|^{2 l}|\hat{h}(\xi)|^{2}\right) \int_{|\xi| \leq 1}|\xi|^{2(k-l)} e^{-c|\xi|^{2} t} d \xi \\
& \leq C(1+t)^{-\frac{d}{2}-(k-l)}\left\|\partial_{x}^{l} h\right\|_{L^{1}}^{2} .
\end{aligned}
$$


On the other hand, since $\rho(\xi) \geq \frac{1}{2}$ for $|\xi| \geq 1$, we have

$$
\int_{|\xi| \geq 1}|\xi|^{2 k} e^{-c \rho(\xi) t}|\hat{h}(\xi)|^{2} d \xi \leq e^{-c t} \int_{|\xi| \geq 1}|\xi|^{2 k}|\hat{h}(\xi)|^{2} d \xi \leq e^{-c t}\left\|\partial_{x}^{k} h\right\|_{L^{2}}^{2},
$$

where the Plancherel theorem has been used again. The last two inequalities give (5.10) and hence the proof is complete.

Next, we introduce the semigroup $e^{t \Phi}$ associated with the linear problem (5.1). According to (5.4), this semigroup can be represented with the inverse Fourier transform as

$$
\left(e^{t \Phi} h\right)(x)=(2 \pi)^{-\frac{d}{2}} \int_{\mathbb{R}_{\xi}^{d}} e^{t \Phi(i \xi)} \hat{h}(\xi) e^{i \xi \cdot x} d \xi
$$

with

$$
\Phi(i \xi)=-\left(A^{0}\right)^{-1}(A(i \xi)+L)
$$

where $A(i \xi)=i \sum_{j=1}^{d} A^{j} \xi_{j}$.

Note that $z(x, t):=\left(e^{t \Phi} h\right)(x)$ is a solution to (5.1) with $p=0, q=0$ and with the initial data $h(x)$. Therefore it follows from Proposition 5.1 that $\left|e^{t \Phi(i \xi)}\right| \leq C e^{-c \rho(\xi) t}$. Also, as a corollary of Lemma 5.2 we have the following decay estimate for the semigroup $e^{t \Phi}$.

Lemma 5.3. Under the conditions of Theorem 3.2, it holds that

$$
\left\|\partial_{x}^{k} e^{t \Phi} h\right\|_{L^{2}} \leq C e^{-c t}\left\|\partial_{x}^{k} h\right\|_{L^{2}}+C(1+t)^{-\frac{d}{4}-\frac{1}{2}(k-l)}\left\|\partial_{x}^{l} q\right\|_{L^{1}}
$$

for any non-negative integers $k$ and $l$ with $l \leq k$.

In case $d=1$ and $h=\left(A^{0}\right)^{-1} q$ with $q \in \mathcal{M}^{\perp}$, this lemma can be improved as follows.

Lemma 5.4. Let $d=1$. Under the conditions of Theorem 3.2, if $q(x) \in \mathcal{M}^{\perp}$ for $x \in \mathbb{R}^{d}$, then it holds that

$$
\left\|\partial_{x}^{k} e^{t \Phi}\left(A^{0}\right)^{-1} q\right\|_{L^{2}} \leq C e^{-c t}\left\|\partial_{x}^{k} q\right\|_{L^{2}}+C(1+t)^{-\frac{3}{4}-\frac{1}{2}(k-l)}\left\|\partial_{x}^{l} q\right\|_{L^{1}}
$$

for any non-negative integers $k$ and $l$ with $l \leq k+1$.

Proof. Since $q \in \mathcal{M}^{\perp}$, by Lemma A.2 in Appendix there is a positive constant $r_{0}$ such that $\left|e^{t \Phi(i \xi)}\left(A^{0}\right)^{-1} \hat{q}(\xi)\right| \leq C e^{-c t}|\hat{q}(\xi)|+C|\xi| e^{-c \xi^{2} t}|\hat{q}(\xi)|$ for $|\xi| \leq r_{0}$. Thus, as in the proof of the inequality (5.10), we have

$$
\begin{aligned}
& \int_{|\xi| \leq r_{0}}|\xi|^{2 k}\left|e^{t \Phi(i \xi)}\left(A^{0}\right)^{-1} \hat{q}(\xi)\right|^{2} d \xi \\
& \leq C e^{-c t} \int_{|\xi| \leq r_{0}}|\xi|^{2 k}|\hat{q}(\xi)|^{2} d \xi+C \int_{|\xi| \leq r_{0}}|\xi|^{2(k+1)} e^{-c \xi^{2} t}|\hat{q}(\xi)|^{2} d \xi \\
& \leq C e^{-c t}\left\|\partial_{x}^{k} q\right\|_{L^{2}}^{2}+C(1+t)^{-\frac{1}{2}-(k+1-l)}\left\|\partial_{x}^{l} q\right\|_{L^{1}}^{2} .
\end{aligned}
$$


On the other hand, since $\left|e^{t \Phi(i \xi)}\right| \leq C e^{-c t}$ for $|\xi| \geq r_{0}$, we have

$$
\int_{|\xi| \geq r_{0}}|\xi|^{2 k}\left|e^{t \Phi(i \xi)}\left(A^{0}\right)^{-1} \hat{q}(\xi)\right|^{2} d \xi \leq e^{-c t}\left\|\partial_{x}^{k} q\right\|_{L^{2}}^{2}
$$

These estimates together with the Plancherel theorem prove the lemma.

\section{Multi-dimensional nonlinear problems}

In this section, we use Lemma 5.2 to derive sharper decay estimates of solutions to the multi-dimensional nonlinear problem (1.1) under the additional condition $w_{0}(x)-\bar{w} \in H^{s} \cap L^{1}$.

Set $w=\bar{w}+D_{u} w(\bar{u}) z$. The nonlinear system (1.1) can be rewritten as

$$
A^{0} z_{t}+\sum_{j=1}^{d} A^{j} z_{x_{j}}+L z=\sum_{j=1}^{d} p_{x_{j}}^{j}+q
$$

where $A^{0}=A^{0}(\bar{u}), A^{j}=A^{j}(\bar{u}), L=L(\bar{u})$, and

$$
\begin{aligned}
p^{j} & =-\left\{f^{j}(w)-f^{j}(\bar{w})-D_{w} f^{j}(\bar{w})(w-\bar{w})\right\} \\
q & =g(w)-g(\bar{w})-D_{w} g(\bar{w})(w-\bar{w}) .
\end{aligned}
$$

For these coefficient matrices, see (2.3) and (2.4). Note that $q \in \mathcal{M}^{\perp}$. Also, we have from the calculus inequalities in Sobolev spaces that

$$
\begin{aligned}
\left\|\partial_{x}^{k} p\right\|_{L^{1}} & \leq C\|w-\bar{w}\|_{L^{2}}\left\|\partial_{x}^{k}(w-\bar{w})\right\|_{L^{2}} \\
\left\|\partial_{x}^{k} p\right\|_{L^{2}} & \leq C\|w-\bar{w}\|_{L^{\infty}}\left\|\partial_{x}^{k}(w-\bar{w})\right\|_{L^{2}}
\end{aligned}
$$

whenever $w=w(x, t)$ takes values in a neighborhood $\bar{w} \in \mathcal{E}$, where $0 \leq k \leq s$. The same estimates hold true also for $q$.

To show the decay estimates, we introduce

$$
N(t):=\sum_{k=0}^{s-1} \sup _{0 \leq \tau \leq t}(1+\tau)^{\frac{d}{4}+\frac{k}{2}}\left\|\partial_{x}^{k}(w(\tau)-\bar{w})\right\|_{L^{2}} .
$$

Note that the Gagliardo-Nirenberg inequality (4.14) gives

$$
\|w(t)-\bar{w}\|_{L^{\infty}} \leq C N(t)(1+t)^{-\frac{d}{2}} .
$$

Moreover, from (6.2) we deduce that

$$
\begin{aligned}
\left\|\partial_{x}^{k} p(t)\right\|_{L^{1}} & \leq C N(t)^{2}(1+t)^{-\frac{d}{2}-\frac{k}{2}} \\
\left\|\partial_{x}^{k} p(t)\right\|_{L^{2}} & \leq C N(t)^{2}(1+t)^{-\frac{3 d}{4}-\frac{k}{2}}
\end{aligned}
$$


for $0 \leq k \leq s-1$. The same estimates hold true also for $q$. In addition, we have

$$
\left\|\partial_{x}^{k} p(t)\right\|_{H^{1}} \leq C E(t) N(t)(1+t)^{-\frac{d}{2}-\frac{k}{2}}
$$

for $0 \leq k \leq s-1$, where $E(t)$ is defined in (4.1) with $v$ replaced by $w$. Here we have used the fact that $\left\|\partial_{x}^{k} p\right\|_{H^{1}} \leq C\|w-\bar{w}\|_{L^{\infty}}\left\|\partial_{x}^{k}(w-\bar{w})\right\|_{H^{1}}$ and $\left\|\partial_{x}^{k}(w(t)-\bar{w})\right\|_{H^{1}} \leq E(t)(1+t)^{-\frac{k}{2}}$. Note that $N(t)$ does not control $\left\|\partial_{x}^{s} w(t)\right\|_{L^{2}}$ and therefore $E(t)$ is used in (6.6).

With these preparations, we apply Lemma 5.2 to (6.1) and obtain the following inequality on $N(t)$.

Proposition 6.1. Let $d \geq 2$ and $s>\frac{d}{2}+1$. Under the conditions of Theorem 3.2, suppose that $w_{0}-\bar{w} \in H^{s} \cap L^{1}$ and put $E_{1}:=\left\|w_{0}-\bar{w}\right\|_{H^{s}}+\left\|w_{0}-\bar{w}\right\|_{L^{1}}$. Then it holds that

$$
N(t) \leq C E_{1}+C N(t)^{2}+C E(t) N(t) .
$$

Proof. Applying Lemma 5.2 to (6.1) gives

$$
\begin{aligned}
\left\|\partial_{x}^{k}(w(t)-\bar{w})\right\|_{L^{2}}^{2} \leq & C e^{-c t}\left\|\partial_{x}^{k}\left(w_{0}-\bar{w}\right)\right\|_{L^{2}}^{2}+C(1+t)^{-\frac{d}{2}-k}\left\|w_{0}-\bar{w}\right\|_{L^{1}}^{2} \\
& +C \int_{0}^{t} e^{-c(t-\tau)}\left(\left\|\partial_{x}^{k} p(\tau)\right\|_{H^{1}}^{2}+\left\|\partial_{x}^{k} q(\tau)\right\|_{L^{2}}^{2}\right) d \tau \\
& +C \int_{0}^{\frac{t}{2}}(1+t-\tau)^{-\frac{d}{2}-k}\left(\|p(\tau)\|_{L^{1}}^{2}+\|q(\tau)\|_{L^{1}}^{2}\right) d \tau \\
& +C \int_{\frac{t}{2}}^{t}(1+t-\tau)^{-\frac{d}{2}}\left(\left\|\partial_{x}^{k} p(\tau)\right\|_{L^{1}}^{2}+\left\|\partial_{x}^{k} q(\tau)\right\|_{L^{1}}^{2}\right) d \tau
\end{aligned}
$$

for $0 \leq k \leq s-1$. For the integrals on the right-hand side of (6.8), we use the inequalities in (6.5) and (6.6) to estimate them as follows. It is clear that

$$
\begin{aligned}
& \int_{0}^{t} e^{-c(t-\tau)}\left(\left\|\partial_{x}^{k} p(\tau)\right\|_{H^{1}}^{2}+\left\|\partial_{x}^{k} q(\tau)\right\|_{L^{2}}^{2}\right) d \tau \\
& \leq C E(t)^{2} N(t)^{2} \int_{0}^{t} e^{-c(t-\tau)}(1+\tau)^{-d-k} d \tau+C N(t)^{4} \int_{0}^{t} e^{-c(t-\tau)}(1+\tau)^{-3 \frac{d}{2}-k} d \tau \\
& \leq C E(t)^{2} N(t)^{2}(1+t)^{-d-k}+C N(t)^{4}(1+t)^{-\frac{3 d}{2}-k} .
\end{aligned}
$$

Moreover, since $d \geq 2$, we have

$$
\begin{aligned}
\int_{0}^{\frac{t}{2}}(1+t-\tau)^{-\frac{d}{2}-k} & \left(\|p(\tau)\|_{L^{1}}^{2}+\|q(\tau)\|_{L^{1}}^{2}\right) d \tau \\
& \leq C N(t)^{4} \int_{0}^{\frac{t}{2}}(1+t-\tau)^{-\frac{d}{2}-k}(1+\tau)^{-d} d \tau \\
& \leq C N(t)^{4}(1+t)^{-\frac{d}{2}-k}
\end{aligned}
$$


and

$$
\begin{aligned}
\int_{\frac{t}{2}}^{t}(1+t-\tau)^{-\frac{d}{2}} & \left(\left\|\partial_{x}^{k} p(\tau)\right\|_{L^{1}}^{2}+\left\|\partial_{x}^{k} q(\tau)\right\|_{L^{1}}^{2}\right) d \tau \\
& \leq C N(t)^{4} \int_{\frac{t}{2}}^{t}(1+t-\tau)^{-\frac{d}{2}}(1+\tau)^{-d-k} d \tau \\
& \leq C N(t)^{4} \vartheta(t)(1+t)^{-d-k} \\
& \leq C N(t)^{4}(1+t)^{-\frac{d}{2}-k}
\end{aligned}
$$

where $\vartheta(t)=\log (1+t)$ if $d=2$ and $\vartheta(t)=1$ for $d \geq 3$. Substituting all the last three estimates into (6.8), we obtain

$$
(1+t)^{\frac{d}{2}+k}\left\|\partial_{x}^{k}(w(t)-\bar{w})\right\|_{L^{2}}^{2} \leq C E_{1}^{2}+C N(t)^{4}+C E(t)^{2} N(t)^{2}
$$

for $0 \leq k \leq s-1$. Summing up this inequality for $k$ gives $N(t)^{2} \leq C E_{1}^{2}+$ $C N(t)^{2}+C E(t)^{2} N(t)^{2}$, which is equivalent to (6.7). This completes the proof.

Having Proposition 6.1, we turn to prove the main result of this section.

Theorem 6.2. Let $d \geq 2$ and $s>\frac{d}{2}+1$. Under the conditions of Theorem 3.2, suppose $w_{0}-\bar{w} \in H^{s} \cap L^{1}$ and $E_{1}$ is sufficiently small. Then the global solution $w(x, t)$ to (1.1) satisfies the following decay estimates:

$$
\left\|\partial_{x}^{k}(w(t)-\bar{w})\right\|_{L^{p}} \leq C E_{1}(1+t)^{-\gamma_{p}-\frac{k}{2}}
$$

for $p \in[2, \infty]$ and $0 \leq k \leq s-1-2 \gamma_{p}^{\prime}\left(k \neq s-1-\frac{d}{2}\right.$ if $\left.p=\infty\right)$, and

$$
\left\|(I-P) \partial_{x}^{k} v(t)\right\|_{L^{p}} \leq C E_{1}(1+t)^{-\gamma_{p}-\frac{1}{2}(k+1)}
$$

for $p \in[2, \infty]$ and $0 \leq k \leq s-2-2 \gamma_{p}^{\prime}\left(k \neq s-2-\frac{d}{2}\right.$ if $\left.p=\infty\right)$. Here $\gamma_{p}=\frac{d}{2}\left(1-\frac{1}{p}\right)$ is the decay exponent of the $L^{p}-L^{1}$ decay estimate for the linear heat equation and $\gamma_{p}^{\prime}=\frac{d}{2}\left(\frac{1}{2}-\frac{1}{p}\right)$ is the same as in Theorem 4.3 .

Proof. From Theorem 4.3 we see that $E(t) \leq C\left\|w_{0}-\bar{w}\right\|_{H^{s}} \leq C E_{1}$. Thus, if $E_{1}$ is sufficiently small, we see from (6.7) that $N(t) \leq C E_{1}+C N(t)^{2}$, which implies that $N(t) \leq C E_{1}$, provided that $E_{1}$ is sufficiently small. Consequently, we obtain the decay estimate (6.9) for $p=2$ and $0 \leq k \leq s-1$. Obviously, this gives

$$
\left\|\partial_{x}^{k}(v(t)-\bar{v})\right\|_{L^{2}} \leq C E_{1}(1+t)^{-\frac{d}{4}-\frac{k}{2}}
$$

for $0 \leq k \leq s-1$. Moreover, a direct use of the Gagliardo-Nirenberg inequality (4.16) leads to (6.9). 
To show (6.10), we look back at the proof of Proposition 4.2. We simplify (4.13) as

$$
\left\|\partial_{x}^{k} \tilde{b}_{2}\right\|_{L^{2}} \leq C\left\|\partial_{x}^{k+1} v\right\|_{L^{2}}+C\|v-\bar{v}\|_{L^{\infty}}\left\|\partial_{x}^{k}(v-\bar{v})\right\|_{L^{2}}
$$

for $0 \leq k \leq s-1$. Substituting (6.4) and (6.11), we obtain $\left\|\partial_{x}^{k} \tilde{b}_{2}(t)\right\|_{L^{2}} \leq$ $C E_{1}(1+t)^{-\frac{d}{4}-\frac{1}{2}(k+1)}$ for $0 \leq k \leq s-2$. Consequently, we have

$$
\begin{aligned}
\int_{0}^{t} e^{-c(t-\tau)}\left\|\partial_{x}^{k} \tilde{b}_{2}(\tau)\right\|_{L^{2}} d \tau & \leq C E_{1} \int_{0}^{t} e^{-c(t-\tau)}(1+\tau)^{-\frac{d}{4}-\frac{1}{2}(k+1)} d \tau \\
& \leq C E_{1}(1+t)^{-\frac{d}{4}-\frac{1}{2}(k+1)}
\end{aligned}
$$

for $0 \leq k \leq s-2$. This, together with (4.12), gives $\left\|(I-P) \partial_{x}^{k} v(t)\right\|_{L^{2}} \leq$ $C E_{1}(1+t)^{-\frac{d}{4}-\frac{1}{2}(k+1)}$ for $0 \leq k \leq s-2$ and, moreover, the desired estimate (6.10) by using the Gagliardo-Nirenberg inequality (4.16). This completes the proof.

\section{One-dimensional nonlinear problems}

In this section, we prove Proposition 6.1 and Theorem 6.2 for one-dimensional nonlinear problem (6.1):

$$
A^{0} z_{t}+A z_{x}+L z=p_{x}+q
$$

with $A=A^{1}(\bar{u})$ and $p=p^{1}$. Although the two hold true even for $d=1$, the proofs in the previous section are not valid for $d=1$. In particular, we will prove Theorem 6.2 under the additional regularity $s \geq 3$. Also, we will use Lemmas 5.3 and 5.4, instead of Lemma 5.2, to prove Proposition 6.1. Namely,

Proposition 7.1. Let $d=1$ and $s \geq 2$. Under the conditions of Theorem 3.2, suppose that $w_{0}-\bar{w} \in H^{s} \cap L^{1}$. Then it holds that

$$
N(t) \leq C E_{1}+C N(t)^{2}+C E(t) N(t)
$$

Proof. Using the semigroup $e^{t \Phi}$ associated with the linearized system (7.1), the solution $z=z(x, t)$ to $(7.1)$ can be represented as $z(t)=e^{t \Phi} z_{0}+\int_{0}^{t} e^{(t-\tau) \Phi}\left(A^{0}\right)^{-1}$. $\left(p_{x}+q\right)(\tau) d \tau$. From this representation we have

$$
\begin{aligned}
\left\|\partial_{x}^{k}(w(t)-\bar{w})\right\|_{L^{2}} \leq & C\left\|\partial_{x}^{k} e^{t \Phi}\left(w_{0}-\bar{w}\right)\right\|_{L^{2}}+C \int_{0}^{t}\left\|\partial_{x}^{k+1} e^{(t-\tau) \Phi}\left(A^{0}\right)^{-1} p(\tau)\right\|_{L^{2}} d \tau \\
& +C \int_{0}^{t}\left\|\partial_{x}^{k} e^{(t-\tau) \Phi}\left(A^{0}\right)^{-1} q(\tau)\right\|_{L^{2}} d \tau
\end{aligned}
$$


for $0 \leq k \leq s-1$. Moreover, we note that $q \in \mathcal{M}^{\perp}$ and deduce from Lemmas 5.3 and 5.4 that

$$
\begin{aligned}
\left\|\partial_{x}^{k}(w(t)-\bar{w})\right\|_{L^{2}} \leq & C e^{-c t}\left\|\partial_{x}^{k}\left(w_{0}-\bar{w}\right)\right\|_{L^{2}}+C(1+t)^{-\frac{1}{4}-\frac{k}{2}}\left\|w_{0}-\bar{w}\right\|_{L^{1}} \\
& +C \int_{0}^{t} e^{-c(t-\tau)}\left(\left\|\partial_{x}^{k+1} p(\tau)\right\|_{L^{2}}+\left\|\partial_{x}^{k} q(\tau)\right\|_{L^{2}}\right) d \tau \\
& +C \int_{0}^{\frac{t}{2}}(1+t-\tau)^{-\frac{3}{4}-\frac{k}{2}}\left(\|p(\tau)\|_{L^{1}}+\|q(\tau)\|_{L^{1}}\right) d \tau \\
& +C \int_{\frac{t}{2}}^{t}(1+t-\tau)^{-\frac{3}{4}}\left(\left\|\partial_{x}^{k} p(\tau)\right\|_{L^{1}}+\left\|\partial_{x}^{k} q(\tau)\right\|_{L^{1}}\right) d \tau .
\end{aligned}
$$

Now we use the inequalities in (6.5) and (6.6) to estimate each term on the right-hand side of (7.3) as follows. We have

$$
\begin{aligned}
& \int_{0}^{t} e^{-c(t-\tau)}\left(\left\|\partial_{x}^{k+1} p(\tau)\right\|_{L^{2}}+\left\|\partial_{x}^{k} q(\tau)\right\|_{L^{2}}\right) d \tau \\
& \leq C E(t) N(t) \int_{0}^{t} e^{-c(t-\tau)}(1+\tau)^{-\frac{1}{2}-\frac{k}{2}} d \tau+C N(t)^{2} \int_{0}^{t} e^{-c(t-\tau)}(1+\tau)^{-\frac{3}{4}-\frac{k}{2}} d \tau \\
& \leq C E(t) N(t)(1+t)^{-\frac{1}{2}-\frac{k}{2}}+C N(t)^{2}(1+t)^{-\frac{3}{4}-\frac{k}{2}}, \\
& \int_{0}^{\frac{t}{2}}(1+t-\tau)^{-\frac{3}{4}-\frac{k}{2}}\left(\|p(\tau)\|_{L^{1}}+\|q(\tau)\|_{L^{1}}\right) d \tau \\
& \leq C N(t)^{2} \int_{0}^{\frac{t}{2}}(1+t-\tau)^{-\frac{3}{4}-\frac{k}{2}}(1+\tau)^{-\frac{1}{2}} d \tau \\
& \leq C N(t)^{2}(1+t)^{-\frac{1}{4}-\frac{k}{2}}
\end{aligned}
$$

and

$$
\begin{aligned}
\int_{\frac{t}{2}}^{t}(1+t-\tau)^{-\frac{3}{4}} & \left(\left\|\partial_{x}^{k} p(\tau)\right\|_{L^{1}}^{2}+\left\|\partial_{x}^{k} q(\tau)\right\|_{L^{1}}^{2}\right) d \tau \\
& \leq C N(t)^{2} \int_{\frac{t}{2}}^{t}(1+t-\tau)^{-\frac{3}{4}}(1+\tau)^{-\frac{1}{2}-\frac{k}{2}} d \tau \\
& \leq C N(t)^{2}(1+t)^{-\frac{1}{4}-\frac{k}{2}}
\end{aligned}
$$

Thus we obtain $(1+t)^{\frac{1}{4}+\frac{k}{2}}\left\|\partial_{x}^{k}(w(t)-\bar{w})\right\|_{L^{2}} \leq C E_{1}+C N(t)^{2}+C E(t) N(t)$ for $0 \leq k \leq s-1$. Summing up this inequality for $0 \leq k \leq s-1$, we arrive at the desired inequality (7.2). This completes the proof.

Notice that Theorem 4.3 has no counterpart for $d=1$ because (4.15) fails for $d=1$. Thus, to control $E(t)$ in (7.2), we introduce

$$
N_{\perp}(t):=\sum_{k=0}^{s-2} \sup _{0 \leq \tau \leq t}(1+\tau)^{\frac{3}{4}+\frac{k}{2}}\left\|(I-P) \partial_{x}^{k} v(\tau)\right\|_{L^{2}}
$$


For this time-weighted quantity, we have the following analogue of Proposition 4.2:

Proposition 7.2. Let $d=1$ and $s \geq 2$. The conditions of Proposition 4.2 imply that

$$
N_{\perp}(t) \leq C\left\|v_{0}-\bar{v}\right\|_{H^{s-2}}+C N(t)+C N(t)^{2},
$$

where $N(t)$ is defined in (6.3) with $w$ replaced by $v$ defined through (2.8).

Proof. Recall the proof of Proposition 4.2. We deduce from (6.12) and (6.4) with $d=1$ that

$$
\begin{aligned}
\int_{0}^{t} e^{-c(t-\tau)}\left\|\partial_{x}^{k} \tilde{b}_{2}(\tau)\right\|_{L^{2}} d \tau & \leq C\left(N(t)+N(t)^{2}\right) \int_{0}^{t} e^{-c(t-\tau)}(1+\tau)^{-\frac{3}{4}-\frac{k}{2}} d \tau \\
& \leq C\left(N(t)+N(t)^{2}\right)(1+t)^{-\frac{3}{4}-\frac{k}{2}}
\end{aligned}
$$

for $0 \leq k \leq s-2$. Substituting this into (4.12), we obtain

$$
(1+t)^{\frac{3}{4}+\frac{k}{2}}\left\|(I-P) \partial_{x}^{k} v(t)\right\|_{L^{2}} \leq C\left\|v_{0}-\bar{v}\right\|_{H^{s-2}}+C\left(N(t)+N(t)^{2}\right)
$$

for $0 \leq k \leq s-2$. Summing up this inequality for $k$ gives the desired inequality (7.4). This completes the proof.

Now we are in a position to state our last main result.

Theorem 7.3. Let $d=1$ and $s \geq 3$. Under the conditions of Theorem 3.2, suppose $w_{0}-\bar{w} \in H^{s} \cap L^{1}$ and $E_{1}$ is sufficiently small. Then the global solution $w(x, t)$ to (1.1) satisfies the following decay estimates

$$
\left\|\partial_{x}^{k}(w(t)-\bar{w})\right\|_{L^{p}} \leq C E_{1}(1+t)^{-\gamma_{p}-\frac{k}{2}}
$$

for $p \in[2, \infty]$ and $0 \leq k \leq s-1-2 \gamma_{p}^{\prime}$, and

$$
\left\|(I-P) \partial_{x}^{k} v(t)\right\|_{L^{p}} \leq C E_{1}(1+t)^{-\gamma_{p}-\frac{1}{2}(k+1)}
$$

for $p \in[2, \infty]$ and $0 \leq k \leq s-2-2 \gamma_{p}^{\prime}$. Here $\gamma_{p}=\frac{1}{2}\left(1-\frac{1}{p}\right)$ and $\gamma_{p}^{\prime}=\frac{1}{2}\left(\frac{1}{2}-\frac{1}{p}\right)$ for $d=1$.

Remark 7.4. The regularity $s \geq 2$ is enough for the decay estimate

$$
\|w(t)-\bar{w}\|_{H^{s-1}} \leq C(1+t)^{-\frac{1}{4}}
$$

(see [16]) and for semilinear problems where $p^{1}=0$ (see [8]). 
Proof. Thanks to $s \geq 3$, we deduce from the Gagliardo-Nirenberg inequality (4.14) that

$$
\begin{aligned}
\left\|\partial_{x} v(t)\right\|_{L^{\infty}} & \leq C N(t)(1+t)^{-1} \\
\|(I-P) v(t)\|_{L^{\infty}} & \leq C N_{\perp}(t)(1+t)^{-1} .
\end{aligned}
$$

Thus, in place of (4.15), we have $M(t) \leq C\left(N(t)+N_{\perp}(t)\right)$. Combining this with (4.2), (7.2) and (7.4), we conclude that $N(t)+N_{\perp}(t)+E(t)+D(t) \leq C E_{1}$, provided that $E_{1}$ is sufficiently small. Thus, we obtain

$$
\left\|\partial_{x}^{k}(w(t)-\bar{w})\right\|_{L^{2}} \leq C E_{1}(1+t)^{-\frac{1}{4}-\frac{k}{2}}
$$

for $0 \leq k \leq s-1$, and

$$
\left\|(I-P) \partial_{x}^{k} v(t)\right\|_{L^{2}} \leq C E_{1}(1+t)^{-\frac{3}{4}-\frac{k}{2}}
$$

for $0 \leq k \leq s-2$. Moreover, the desired estimates follow simply from the Gagliardo-Nirenberg inequality (4.16) with $d=1$. This completes the proof.

\section{A. Spectral analysis for one-dimensional linear problems}

The aim here is to prove Lemma A.2 below for one-dimensional problems. In what follows, we set $\Psi(z)=\Lambda \Phi(z) \Lambda^{-1}$ for complex number $z$, where $\Lambda=\left(A^{0}\right)^{\frac{1}{2}}$ is real symmetric and positive definite, and $\Phi(i \xi)=-\left(A^{0}\right)^{-1}(i \xi A+L)$ is defined in $(5.11)$.

Since $\Psi(0)=-\Lambda^{-1} L \Lambda^{-1}$ is real symmetric, its spectral representation is of the form

$$
\Psi(0)=\sum_{j=1}^{J} \lambda_{j} \bar{\Pi}_{j}
$$

where the $\lambda_{j}$ 's are $J$ distinct (real) eigenvalues of $\Psi(0)$ and $\bar{\Pi}_{j}$ is the corresponding eigenprojection. Note that each $\bar{\Pi}_{j}$ is real symmetric and satisfies

$$
\sum_{j=1}^{J} \bar{\Pi}_{j}=I, \quad \bar{\Pi}_{j}^{2}=\bar{\Pi}_{j}, \quad \bar{\Pi}_{j} \bar{\Pi}_{k}=0 \quad \text { for } j \neq k .
$$

Since $\Psi(0)$ is nonpositive definite, without loss of generality, we assume $\lambda_{1}=0$. Then $\bar{\Pi}_{1} \mathbb{R}^{m}$ is the null space of $\Psi(0)$ and therefore

$$
\bar{\Pi}_{1} \mathbb{R}^{m}=\Lambda \mathcal{M} \quad \text { and } \quad \lambda_{j}<0 \quad \text { for } j=2,3, \ldots, J
$$

where $\mathcal{M}$ is the null space of $L$. 
Note that $\Psi(z)$ is analytic with respect to $z$ and is real symmetric for real $z$. According to the perturbation theory for one-parameter family of matrices [6], $\Psi(z)$ has the spectral representation of the form

$$
\Psi(z)=\sum_{l=1}^{\bar{J}} \lambda_{l}(z) \Pi_{l}(z)
$$

for $z$ close to 0 . Here $\bar{J}$ is the number of distinct eigenvalues of $\Psi(z), \lambda_{l}(z)$ is an eigenvalue, and $\Pi_{l}(z)$ is the corresponding eigenprojection. Moreover, $\bar{J}$ is a constant for $z$ close but unequal to $0, \lambda_{l}(z)$ and $\Pi_{l}(z)$ are analytic at $z=0$, each $\Pi_{l}(z)$ is real symmetric for real $z$, and the $\Pi_{l}(z)$ 's satisfy

$$
\sum_{l=1}^{\bar{J}} \Pi_{l}(z)=I, \quad \Pi_{l}(z)^{2}=\Pi_{l}(z), \quad \Pi_{l}(z) \Pi_{k}(z)=0 \quad \text { for } l \neq k .
$$

Letting $z \rightarrow 0$ in (A.2), we have $\Psi(0)=\sum_{l=1}^{\bar{J}} \lambda_{l}(0) \Pi_{l}(0)$. Thus $\lambda_{l}(0)$ is equal to a certain eigenvalue $\lambda_{j}$ of $\Psi(0)$. Let $n_{j}$ be the number of $\lambda_{l}(z)$ satisfying $\lambda_{l}(0)=\lambda_{j}$ and rename the $n_{j}$ eigenvalues $\lambda_{l}(z)$ as $\lambda_{j \alpha}(z)$ with $\alpha=1,2, \ldots, n_{j}$. Then the spectral representation of $\Psi(z)$ can be rewritten as

$$
\Psi(z)=\sum_{j=1}^{J} \sum_{\alpha=1}^{n_{j}} \lambda_{j \alpha}(z) \Pi_{j \alpha}(z)
$$

and (A.3) becomes

$$
\begin{array}{ll}
\sum_{j=1}^{J} \sum_{\alpha=1}^{n_{j}} \Pi_{j \alpha}(z)=I, & \Pi_{j \alpha}(z)^{2}=\Pi_{j \alpha}(z) \\
\Pi_{j \alpha}(z) \Pi_{j^{\prime} \alpha^{\prime}}(z)=0 & \text { for }(j, \alpha) \neq\left(j^{\prime}, \alpha^{\prime}\right) .
\end{array}
$$

Moreover, we have

$$
\sum_{\alpha=1}^{n_{j}} \Pi_{j \alpha}(0)=\bar{\Pi}_{j} \quad \text { and } \quad \lambda_{j \alpha}(0)=\lambda_{j} .
$$

Since $\lambda_{j \alpha}(z)$ and $\Pi_{j \alpha}(z)$ are analytic at $z=0$, they have expansions

$$
\lambda_{j \alpha}(z)=\sum_{k=0}^{\infty} z^{k} \lambda_{j \alpha}^{(k)}, \quad \Pi_{j \alpha}(z)=\sum_{k=0}^{\infty} z^{k} \Pi_{j \alpha}^{(k)} .
$$

Note that $\lambda_{j \alpha}^{(k)}$ and $\Pi_{j \alpha}^{(k)}$ are real, since so are $\lambda_{j \alpha}(z)$ and $\Pi_{j \alpha}(z)$ for real $z$. Thanks to (A.1), we see from (A.6) and (A.5) that

$$
\Pi_{1 \alpha}^{(0)} \mathbb{R}^{m}=\Pi_{1 \alpha}(0) \mathbb{R}^{m} \subset \Lambda \mathcal{M} \quad \text { for } \alpha=1,2, \ldots, n_{1} .
$$

Furthermore, we have 
Lemma A.1. $\lambda_{1 \alpha}^{(2)}>0$ for $\alpha=1,2, \ldots, n_{1}$.

Proof. Fix $\alpha$ and choose $\phi \in \mathbb{R}^{m}$ so that $\Pi_{1 \alpha}(0) \phi \neq 0$. Since $\Pi_{1 \alpha}(z) \phi$ is an eigenvector of $\Psi(z)$ associated with the eigenvalue $\lambda_{1 \alpha}(z)$, we substitute the expansions (A.7) into the equation $\Psi(z) \Pi_{1 \alpha}(z) \phi=\lambda_{1 \alpha}(z) \Pi_{1 \alpha}(z) \phi$ to obtain

$$
\begin{aligned}
& \Psi(0) \Pi_{1 \alpha}^{(0)} \phi=\lambda_{1 \alpha}^{(0)} \Pi_{1 \alpha}^{(0)} \phi=0 \\
& \Psi(0) \Pi_{1 \alpha}^{(1)} \phi+\bar{A} \Pi_{1 \alpha}^{(0)} \phi=\lambda_{1 \alpha}^{(1)} \Pi_{1 \alpha}^{(0)} \phi \\
& \Psi(0) \Pi_{1 \alpha}^{(2)} \phi+\bar{A} \Pi_{1 \alpha}^{(1)} \phi=\lambda_{1 \alpha}^{(1)} \Pi_{1 \alpha}^{(1)} \phi+\lambda_{1 \alpha}^{(2)} \Pi_{1 \alpha}^{(0)} \phi
\end{aligned}
$$

where $\bar{A}:=-\Lambda^{-1} A \Lambda^{-1}$ is real symmetric. Now we look at these three equalities. The first one indicates that the non-zero vector $\Pi_{1 \alpha}(0) \phi=\Pi_{1 \alpha}^{(0)} \phi$ is in the null space of $\Psi(0)$. Thus, it follows from the second equality and the stability condition in Section 2 that $\Pi_{1 \alpha}^{(1)} \phi \neq 0$ is not in the null space of $\Psi(0)$. We take the inner products of $\Pi_{1 \alpha}^{(1)} \phi$ with the second equality and of $\Pi_{1 \alpha}^{(0)} \phi$ with the third one to obtain $\left\langle\Pi_{1 \alpha}^{(1)} \phi, \Psi(0) \Pi_{1 \alpha}^{(1)} \phi\right\rangle=\left\langle\Pi_{1 \alpha}^{(1)} \phi,\left(\lambda_{1 \alpha}^{(1)}-\bar{A}\right) \Pi_{1 \alpha}^{(0)} \phi\right\rangle$ and $0=\left\langle\Pi_{1 \alpha}^{(0)} \phi,\left(\lambda_{1 \alpha}^{(1)}-\bar{A}\right) \Pi_{1 \alpha}^{(1)} \phi\right\rangle+\lambda_{1 \alpha}^{(2)}\left|\Pi_{1 \alpha}^{(0)} \phi\right|^{2}$. Hence we have $\lambda_{1 \alpha}^{(2)}\left|\Pi_{1 \alpha}^{(0)} \phi\right|^{2}=$ $-\left\langle\Pi_{1 \alpha}^{(1)} \phi, \Psi(0) \Pi_{1 \alpha}^{(1)} \phi\right\rangle$, which is strictly positive because $\Pi_{1 \alpha}^{(1)} \phi$ is not in the null space of $\Psi(0)$. This completes the proof.

Having these preparations, we prove the main result of this Appendix.

Lemma A.2. If $\hat{q}(\xi) \in \mathcal{M}^{\perp}$ for $\xi \in \mathbb{R}$, then there are positive constants $r_{0}, c$ and $C$ such that

$$
\left|e^{t \Phi(i \xi)}\left(A^{0}\right)^{-1} \hat{q}(\xi)\right| \leq C e^{-c t}|\hat{q}(\xi)|+C|\xi| e^{-c \xi^{2} t}|\hat{q}(\xi)|
$$

for $|\xi| \leq r_{0}$.

Proof. It follows from (A.4) and (A.5) that

$$
\begin{aligned}
e^{t \Phi(i \xi)}\left(A^{0}\right)^{-1} \hat{q}(\xi) & =\Lambda^{-1} e^{t \Psi(i \xi)} \Lambda^{-1} \hat{q}(\xi) \\
& =\sum_{j=1}^{J} \sum_{\alpha=1}^{n_{j}} e^{\lambda_{j \alpha}(i \xi) t} \Lambda^{-1} \Pi_{j \alpha}(i \xi) \Lambda^{-1} \hat{q}(\xi)
\end{aligned}
$$

For $j=2,3, \ldots, J$, since $\lambda_{j \alpha}(0)<0$ due to (A.1) and (A.6), there is a positive constant $r_{1}$ such that for $|\xi| \leq r_{1}$,

$$
\sum_{j=2}^{J} \sum_{\alpha=1}^{n_{j}}\left|e^{\lambda_{j \alpha}(i \xi) t} \Lambda^{-1} \Pi_{j \alpha}(i \xi) \Lambda^{-1} \hat{q}(\xi)\right| \leq C e^{-c t}|\hat{q}(\xi)| .
$$

On the other hand, since $\Pi_{1 \alpha}(0)$ and $\Lambda$ are real symmetric, we find that

$$
\left\langle\Pi_{1 \alpha}(0) \Lambda^{-1} \hat{q}(\xi), \phi\right\rangle=\left\langle\hat{q}(\xi), \Lambda^{-1} \Pi_{1 \alpha}(0) \phi\right\rangle=0
$$


for any $\phi \in \mathbb{R}^{m}$ and $\xi \in \mathbb{R}$, where we used the fact that $\hat{q}(\xi) \in \mathcal{M}^{\perp}$ and $\Lambda^{-1} \Pi_{1 \alpha}(0) \phi \in \mathcal{M}$ due to (A.8). This shows that $\Pi_{1 \alpha}(0) \Lambda^{-1} \hat{q}(\xi)=0$ for $\xi \in \mathbb{R}$. Also, by virtue of Lemma A.1, we have $\operatorname{Re} \lambda_{1 \alpha}(\xi) \leq-c \xi^{2}$ for $|\xi| \leq r_{0}$, where $r_{0}$ is a positive constant with $r_{0} \leq r_{1}$. Thus, we have

$$
\sum_{\alpha=1}^{n_{1}}\left|e^{\lambda_{1 \alpha}(i \xi) t} \Lambda^{-1} \Pi_{1 \alpha}(i \xi) \Lambda^{-1} \hat{q}(\xi)\right| \leq C|\xi| e^{-c \xi^{2} t}|\hat{q}(\xi)|
$$

for $|\xi| \leq r_{0}$. By plugging this and (A.10) into (A.9), the proof is completed.

\section{References}

[1] Bianchini, S., Hanouzet, B. and Natalini, R., Asymptotic behavior of smooth solutions for partially dissipative hyperbolic systems with a convex entropy. Commun. Pure Appl. Math. 60 (2007), 1559 - 1622.

[2] Dafermos, C. M., Hyperbolic Conservation Laws in Continuum Physics (2nd Ed.). Berlin: Springer 2005.

[3] Hanouzet, B. and Natalini, R., Global existence of smooth solutions for partially dissipative hyperbolic systems with a convex entropy. Arch. Rational Mech. Anal. 169 (2003), 89 - 117.

[4] Hosono, T., Large time behavior of solutions to a dissipative wave equation in higher space dimensions. Preprint, Kyushu Univ., (2006).

[5] Iguchi, T. and Kawashima, S., On space-time decay properties of solutions to hyperbolic-elliptic coupled systems. Hiroshima Math. J. 32 (2002), 229 - 308.

[6] Kato, T., Perturbation Theory for Linear Operators (2nd Ed.). New York: Springer 1976.

[7] Kawashima, S., Global existence and stability of solutions for discrete velocity models of the Boltzmann equation. In: emphRecent Topics in Nonlinear PDE (eds.: M. Mimura et al.). Lecture Notes Numer. Appl. Anal. 6. Tokyo: Kinokuniya 1983, pp. $59-85$.

[8] Kawashima, S., Large-time behavior of solutions of the discrete Boltzmann equation. Commun. Math. Phys. 109 (1987), $563-589$.

[9] Kawashima, S. and Yong, W.-A., Dissipative structure and entropy for hyperbolic systems of balance laws. Arch. Rational Mech. Anal. 174 (2004), $345-364$.

[10] Majda, A., Compressible Fluid Flow and Systems of Conservation Laws in Several Space Variables. Appl. Math. Sci. 53. New York: Springer 1984.

[11] Matsumura, A., An energy method for the equations of motion of comptessible viscous and heat-conductive fluids. MRC Technical Summary Report, Univ. of Wisconsin-Madison, \#2194 (1981). 
[12] Shizuta, Y. and Kawashima, S., Systems of equations of hyperbolic-parabolic type with applications to the discrete Boltzmann equation. Hokkaido Math. J. 14 (1985), $249-275$.

[13] Sideris, T. C., Thomases, B. and Wang, D., Long time behavior of solutions to the 3D compressible Euler equations with damping. Comm. Partial Diff. Eqs. 28 (2003), $795-816$.

[14] Yong, W.-A., Basic aspects of hyperbolic relaxation systems. In: Advances in the Theory of Shock Waves (eds.: H. Freistühler and A. Szepessy), Progress in Nonlinear Differential Equations and Their Applications 47, Boston: Birkhäuser 2001, pp. 259 - 305.

[15] Yong, W.-A., Entropy and global existence for hyperbolic balance laws. Arch. Rational Mech. Anal. 172 (2004), 247 - 266.

[16] Yong, W.-A., Entropy and Global Solutions for Hyperbolic Systems of Balance Laws. Habilitationsschrift, University of Heidelberg 2005.

Received March 21, 2007; revised April 28, 2008 